\title{
The Equivalence of the Logarithmic Sobolev Inequality and the Dobrushin-Shlosman Mixing Condition*
}

\author{
Daniel W. Stroock ${ }^{1}$ and Boguslaw Zegarlinski ${ }^{1,2}$ \\ 1 2-272, Mathematics Department, M.I.T., Cambridge MA 02139, USA \\ ${ }^{2}$ Fakultät für Mathematik, Ruhr-Universität Bochum, Postfach 102 148, W-4630 Bochum 1, \\ FRG
}

Received March 25, 1991

\begin{abstract}
Given a finite range lattice gas with a compact, continuous spin space, it is shown (cf. Theorem 1.2) that a uniform logarithmic Sobolev inequality (cf. 1.4) holds if and only if the Dobrushin-Shlosman mixing condition (cf. 1.5) holds. As a consequence of our considerations, we also show (cf. Theorems 3.2 and 3.6) that these conditions are equivalent to a statement about the uniform rate at which the associated Glauber dynamics tends to equilibrium. In this same direction, we show (cf. Theorem 3.19) that these ideas lead to a surprisingly strong large deviation principle for the occupation time distribution of the Glauber dynamics.
\end{abstract}

\section{Introduction}

In our earlier article [S\&Z], we showed (cf. Remark 3.23 in $[S \& Z]$ ) that the Dobrushin-Shlosman mixing condition (cf. (1.5) below) guarantees that the corresponding (necessarily unique) Gibbs state satisfies a logarithmic Sobolev inequality (cf. (1.1) below) and asserted that we would be proving a converse statement in a forthcoming article. This is that "forthcoming article," and the promised converse is the content of Theorem 1.2 below. Because it is difficult to give precise statements of our results before we have introduced the notation explained in Sect. 1 below, we will confine our discussion in this introduction to a few general remarks of a somewhat historical nature. In particular, all that we hope to explain here is the general principle on which our analysis rests.

Ever since the ground-breaking work of Dobrushin, Lanford and Ruelle, most of the analysis of Ising-type models has concentrated on the associated Gibbs

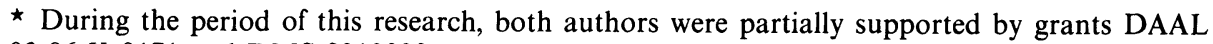
03-86-K-0171 and DMS-8913328 
states and, as such, has been a study of "the equilibrium theory." Unquestionably, the most important goal of this type of analysis has been the determination of when there is only one Gibbs state (i.e., phase transition is absent). In some special cases (e.g., for the classical Ising model itself) great progress has been made toward this goal by the careful application of often deep and intricate combinatorial techniques. Although, at least to date, truly refined results of this sort depend extremely heavily on special properties of the model under consideration, Dobrushin nonetheless introduced in [D] a quite widely applicable criterion (known ever since as the Dobrushin uniqueness condition) which guarantees that a potential admits only one Gibbs state. Although Dobrushin's condition is in some ways quite crude, it is sufficiently strong to verify that, for a large class of potentials (including shift-invariant ones with finite range), uniqueness holds at sufficiently high temperature. In fact, as Gross showed in [G, 2], so long as Dobrushin's condition holds, the mapping taking a potential to its associated Gibbs state is twice differentiable. In this same direction, Dobrushin and Shlosman showed (cf. Theorem 2.1 in [Dob\&S, 1]) that Dobrushin's original uniqueness condition, which was stated in terms of the single site conditional distributions, has a much more general multi-site analogue (condition " $C_{V}$ " in Theorem 2.1 of [Dob\& S, 1]) which also guarantees uniqueness. This condition $C_{V}$ has sometimes been called the Dobrushin-Shlosman uniqueness condition. However, we prefer the authors' original designation $C_{V}$ because of the considerable confusion resulting from the multiplicity of subtly distinct conditions to which the term "Dobrushin-Shlosman uniqueness condition" might be justifiably applied. As distinguished from its predecessor (i.e., Dobrushin's condition), Dobrushin and Shlosman claim in [Dob\&S, 1] that "meditations on Ĉzech models" by Shlosman reveal that $C_{V}$ does not imply that the Gibbs state depends analytically on its potential; and, with this in mind, they initiated the program carried out in [Dob\&S, 1-3], where they introduce some twelve equivalent conditions guaranteeing analytic dependence; and among these twelve is the one which we have chosen to call their mixing condition.

In the preceding paragraph we summarized a small piece of the recent history of the equilibrium theory for Ising-type models. In this paragraph we will give an even briefer summary of a few results about the corresponding dynamical theory (i.e., the dynamics for which Gibbs states are equilibria); for a beautiful and thorough account, see Liggett's book [L]. These dynamical systems (often called Glauber dynamics) are Markov processes which have the following two essential properties: they are completely determined by the given potential and their reversing states (i.e., the initial distributions for which they are reversible) coincides with the set of Gibbs states for the given potential. In particular, if the Glauber dynamics is ergodic in the sense that it admits only one invariant measure (and therefore only one which is reversing), then the corresponding potential admits only one Gibbs state. Perhaps more significant for us here is the observation made in $[\mathrm{H} \& \mathrm{~S}, 1]$ that sufficiently strong convergence of an ergodic Glauber dynamics to its equilibrium allows one to transfer rather obvious mixing properties of the dynamics to the associated Gibbs state. That is, the Glauber dynamics are run in such a way that the probability of two distant sites "communicating" during a finite time interval is exponentially small in the ratio of the distance between them to the length of the time interval; and, therefore, if the dynamics is equilibrating 
sufficiently fast, then this inability to "communicate" becomes a mixing property for the equilibrium measure.

The structure of this paper is the following. In Sect. 1 we introduce the notation, state the main result (cf. Theorem 1.2), and prove an abstract lemma (cf. Lemma 1.8) which formalizes the principle outlined in the preceding paragraph. Next, basing our reasoning on this principle and borrowing from the ideas in [H\&S, 2], we give in Sect. 2 the proof of our main result. Finally, in Sect. 3 we derive a few related results which follow from the same line of reasoning.

\section{Setting, Statements, and Preliminaries}

The basic setting will be the same as that in [S\&Z]. Thus, we will be working in the following context.

The Lattice. The lattice underlying our model will be the $d$-dimensional square lattice $\mathbb{Z}^{d}$ for some fixed $d \in \mathbb{Z}^{+}$, and, for $\mathbf{k}=\left(k^{1}, \ldots, k^{d}\right) \in \mathbb{Z}^{d}$, we will use the norm $|\mathbf{k}| \equiv \max \left|k^{i}\right|$. Given $\Lambda \subseteq \mathbb{Z}^{d}$, we will use $\Lambda \mathrm{C} \equiv \mathbb{Z}^{d} \backslash \Lambda$ to denote the complement $1 \leqq i \leqq d$

of $\Lambda,|\Lambda|$ to denote the cardinality of $\Lambda$, and $\mathbf{k}+\Lambda$ to denote the translate $\{\mathbf{k}+\mathbf{j}: \mathbf{j} \in \Lambda\}$ of $\Lambda$ by $\mathbf{k} \in \mathbb{Z}^{d}$. Furthermore, each $R \in \mathbb{Z}^{+}$, we take the $R$-boundary $\partial_{R} \Lambda$ to be the set

$$
\{\mathbf{k} \in \Lambda \mathrm{C}:|\mathbf{k}-\mathbf{j}| \leqq R \text { for some } \mathbf{j} \in \Lambda\} \text {. }
$$

Finally, we will occasionally use the notation $\Lambda \subset \subset \mathbb{Z}^{d}$ to mean that $|\Lambda|<\infty$, and If will stand for the set of all non-empty $\Lambda \subset \subset \mathbb{Z}^{d}$.

The Spin Space. The spin space for our model will be the compact, connected, finite dimensional, $C^{\infty}$ manifold $M$ equipped with Riemannian metric $(\cdot \mid \cdot)$. Also, we use $\mathbf{T}(M)$ to denote the tangent bundle over $M$ and introduce the associated gradient operator $\nabla: C^{1}(M) \rightarrow C(\mathbf{T}(M))$ and divergence operator div on $C^{1}$-sections of $\mathbf{T}(M)$ onto $C(M)$. Finally, $\mathscr{B}_{M}$ will stand for the Borel field over $M$ and $\lambda$ will be the normalized Riemannian measure on $\left(M, \mathscr{B}_{M}\right)$.

The Configuration Space. Our configuration space will be the product space $\mathbf{M} \equiv M^{\mathbb{Z}^{d}}$ endowed with the product topology. Given a non-empty $\Lambda \subseteq \mathbb{Z}^{d}$, we will use $\mathbf{x} \in \mathbf{M} \mapsto \mathbf{x}_{\Lambda} \in M^{\Lambda}$ to denote the natural projection taking $\mathbf{M}$ onto $M^{\Lambda}, B_{\Lambda}(\mathbf{M})$ and $C_{\Lambda}(\mathbf{M})$ to denote the set of functions on $\mathbf{M}$ of the form $\mathbf{x} \in \mathbf{M} \mapsto \varphi\left(\mathbf{x}_{\Lambda}\right) \in \mathbb{R}$ as $\varphi$ runs over, respectively, the set $B\left(M^{\Lambda}\right)$ of bounded, $\mathscr{B}_{M}^{\Lambda}$-measurable and the set $C\left(M^{\Lambda}\right)$ of continuous functions on $M^{\Lambda}$; and $\mathscr{F}_{\Lambda}$ to denote the $\sigma$-algebra over $\mathbf{M}$ generated by elements of $B_{\Lambda}(\mathbf{M})$. When $\Lambda=\{\mathbf{k}\}$, we will use $x_{\mathbf{k}}$ in place of $\mathbf{x}_{\{\mathbf{k}\}}$; and when $\Lambda=\mathbb{Z}^{d}$, it is clear that $\mathscr{F}_{\Lambda}$ is precisely the Borel field $\mathscr{B}_{\mathbf{M}}$ over $\mathbf{M}$, and we will simply write $\mathscr{F}$ instead of $\mathscr{F}_{\mathbb{Z}^{d}}$. Also, we will say that $f: \mathbf{M} \rightarrow \mathbb{R}$ is local if it is an element of $B_{\Lambda}(\mathbf{M})$ for some $\Lambda \in \mathfrak{F}$. Finally, for each $\mathbf{k} \in \mathbb{Z}^{d}$, we define the shift transformation $\theta^{\mathbf{k}}: \mathbf{M} \rightarrow \mathbf{M}$ so that $\left(\theta^{\mathbf{k}} \mathbf{x}\right)_{\mathbf{j}}=x_{\mathbf{k}+\mathbf{j}}$ for every $\mathbf{x} \in \mathbf{M}$ and every $\mathbf{j} \in \mathbb{Z}^{d}$.

In order to describe a differentiable structure on $\mathbf{M}$, it will be convenient to introduce additional notation. In the first place, given $\varnothing \neq \Lambda \subset \mathbb{Z}^{d}$, we define

$$
\left(\mathbf{x}^{\Lambda}, \mathbf{y}^{\Lambda \mathrm{C}}\right) \in M^{\Lambda} \times M^{\Lambda \mathrm{C}} \mapsto \mathrm{x}^{\Lambda} \cdot \mathbf{y}^{\Lambda \mathrm{C}} \in M
$$


so that $\mathbf{x}^{\Lambda} \cdot \mathbf{y}^{\Lambda \mathrm{C}}$ is the element $\mathbf{z} \in \mathbf{M}$ determined by

$$
\mathbf{z}_{\Lambda}=\mathbf{x}^{\Lambda} \text { and } \mathbf{z}_{\Lambda \mathrm{C}}=\mathbf{y}^{\Lambda \mathrm{C}}
$$

and, for $f: \mathbf{M} \rightarrow \mathbb{R}$ and $\mathbf{y}^{\Lambda \mathrm{C}} \in M^{\Lambda \mathrm{C}}$, we define $f\left(\cdot \mid \mathbf{y}^{\Lambda \mathrm{C}}\right)$ on $M^{\Lambda}$ and $f_{\Lambda}\left(\cdot \mid \mathbf{y}^{\Lambda \mathrm{C}}\right)$ on $\mathbf{M}$ by

and

$$
\mathbf{x}^{\Lambda} \in M^{\Lambda} \mapsto f\left(\mathbf{x}^{\Lambda} \mid \mathbf{y}^{\Lambda C}\right) \equiv f\left(\mathbf{x}^{\Lambda} \cdot \mathbf{y}^{\Lambda C}\right)
$$

$$
\mathbf{x} \in \mathbf{M} \mapsto f_{\Lambda}\left(\mathbf{x} \mid \mathbf{y}^{\Lambda \mathrm{C}}\right) \equiv f\left(\mathbf{x}_{\Lambda} \cdot \mathbf{y}^{\Lambda \mathrm{C}}\right) .
$$

Secondly, for $\mathbf{y} \in \mathbf{M}$, we write $f_{\Lambda}(\mathbf{x} \mid \mathbf{y})$ instead of $f_{\Lambda}\left(\mathbf{x} \mid \mathbf{y}_{\Lambda c}\right)$; and, when $\Lambda=\{\mathbf{k}\}$ we will use $f_{\mathbf{k}}(\cdot \mid \mathbf{y})$ in place of $f_{\{\mathbf{k}\}}(\cdot \mid \mathbf{y})$. Since both

$$
\left(\mathbf{x}^{\Lambda}, \mathbf{y}^{\Lambda \mathrm{C}}\right) \in M^{\Lambda} \times M^{\Lambda \mathrm{C}} \mapsto \mathbf{x}^{\Lambda} \cdot \mathbf{y}^{\Lambda \mathrm{C}} \in \mathbf{M} \quad \text { and } \quad(\mathbf{x}, \mathbf{y}) \in \mathbf{M}^{2} \mapsto \mathbf{x}_{\Lambda} \cdot \mathbf{y}_{\Lambda \mathcal{C}} \in \mathbf{M}
$$

are continuous maps, all the preceding constructions preserve both continuity and measurability; and we now introduce the differentiable structure on $\mathbf{M}$ so that these constructions will also preserve it. Thus, given $n \in \mathbb{Z}^{+}$and a non-empty $\Lambda \subseteq \mathbb{Z}^{d}$, we say that $f \in B_{\Lambda}(\mathbf{M})$ is $n$-times continuously differentiable and write $f \in C^{n}{ }_{\Lambda}(\mathbf{M})$ if

$$
\mathbf{y} \in \mathbf{M} \mapsto f_{\mathbf{k}}(\cdot \mid \mathbf{y}) \in C^{n}(M) \text { is continuous for every } \mathbf{k} \in \Lambda \text {. }
$$

Further, we set

$$
C_{\Lambda}^{\infty}(\mathbf{M})=\bigcap_{n \in \mathbb{Z}^{+}} C_{\Lambda}^{n}(\mathbf{M})
$$

and when $\Lambda=\mathbb{Z}^{d}$, we drop the subscript $\Lambda$ (i.e., $C^{n}(\mathbf{M})=C_{\mathbb{Z}^{d}}^{n}(\mathbf{M})$ ) and introduce

$$
C_{0}^{n}(\mathbf{M}) \equiv \bigcup\left\{f \in C_{\Lambda}^{n}(\mathbf{M}): \Lambda \in \mathfrak{F}\right\}
$$

Next, given $\mathbf{k} \in \mathbb{Z}^{d}$, we define

$$
\left(\nabla_{\mathbf{k}} f\right)(\mathbf{x})=\left(\nabla f_{\mathbf{k}}(\cdot \mid \mathbf{x})\right)\left(x_{\mathbf{k}}\right),
$$

and $\operatorname{div}_{\mathbf{k}}$ to be the corresponding divergence operation taking $C^{1}$-sections

$$
\mathbf{x} \in \mathbf{M} \mapsto F(\mathbf{x}) \in \mathbf{T}_{x_{\mathbf{k}}}(M)
$$

into $C(\mathbf{M})$. Also, for $f \in C^{1}(\mathbf{M})$ and non-empty $\Lambda \subseteq \mathbb{Z}^{d}$, we define

$$
\mathbf{x} \in \mathbf{M} \mapsto \nabla_{\Lambda} f(\mathbf{x}) \in \prod_{\mathbf{k} \in \Lambda} \mathbf{T}_{x_{\mathbf{k}}}(M) \quad \text { so that } \quad\left(\nabla_{\Lambda} f(\mathbf{x})\right)_{\mathbf{k}}=\left(\nabla_{\mathbf{k}} f\right)(\mathbf{x}) \text { for } \mathbf{k} \in \Lambda \text {. }
$$

(In the preceding, $\mathbf{T}_{x_{\mathbf{k}}}(M)$ denotes the tangent space to $M$ at $x_{\mathbf{k}}$.) In keeping with our earlier conventions, we take $\nabla=\nabla_{\mathbb{Z}^{d}}$ and $\nabla_{\mathbf{k}}=\nabla_{\{\mathbf{k}\}}$. Also,

$$
\left|\nabla_{\Lambda} f\right|^{2}(\mathbf{x}) \equiv \sum_{\mathbf{k} \in \Lambda}\left|\nabla f_{\mathbf{k}}(\cdot \mid \mathbf{x})\right|^{2}\left(x_{\mathbf{k}}\right) \in[0, \infty]
$$

and, when the quantities involved are finite, $\left(\nabla_{\Lambda} f \mid \nabla_{\Lambda} g\right)(\mathbf{x})$ is defined by an obvious application of polarization. Finally, we will use $\|\cdot\|_{u}$ to denote the uniform (i.e., "sup") norm.

Measures on $M^{\Lambda}$ and Logarithmic Sobolev Inequalities. For non-empty $\Lambda \subseteq \mathbb{Z}^{d}$, we use $\mathfrak{M}_{1}\left(M^{\Lambda}\right)$ to denote the space of Borel, probability measures $\mu$ on $\left(M^{\Lambda}, \mathscr{B}_{M}^{\Lambda}\right)$, and, given $\mu \in \mathfrak{M}_{1}\left(M^{\Lambda}\right)$, we will use, depending on the context,

$$
\int_{M^{\Lambda}} f\left(\mathbf{x}^{\Lambda}\right) \mu\left(d \mathbf{x}^{\Lambda}\right), \quad \int f d \mu,\langle f, \mu\rangle, \quad \text { and }\langle f\rangle_{\mu}
$$


all to denote the $\mu$-integral of a $\mu$-integrable function $f: M^{\Lambda} \rightarrow \mathbb{R}$. When we discuss convergence in $\mathfrak{M}_{1}\left(M^{\Lambda}\right)$, the underlying topology will be that of weak convergence, sic: $\left\{\mu_{n}\right\}_{1}^{\infty} \subseteq \mathfrak{M}_{1}\left(M^{\Lambda}\right)$ converges to $\mu$ in $\mathfrak{M}_{1}\left(M^{\Lambda}\right)$, written $\mu_{n} \Rightarrow \mu$, means that $\langle f\rangle_{\mu_{n}} \rightarrow$ $\langle f\rangle_{\mu}$ for every $f \in C\left(M^{\Lambda}\right)$. Furthermore, given $\mu \in \mathfrak{M}_{1}(\mathbf{M})$ and $\varnothing \neq \Lambda \subset \mathbb{Z}^{d}$, we use $\mu_{\Lambda} \in \mathfrak{M}_{1}\left(M^{\Lambda}\right)$ to denote the marginal distribution of $\mathbf{x} \in \mathbf{M} \mapsto \mathbf{x}_{\Lambda} \in M^{\Lambda}$ under $\mu$.

Next, for any non-empty $\Lambda \subseteq \mathbb{Z}^{d}$ and $\mu \in \mathfrak{M}_{1}(\mathbf{M})$, we define the logarithmic Sobolev constant $c(\mu ; \Lambda)$ of $\mu$ on $\Lambda$ to be the smallest $c \in[0, \infty]$ with the property that

$$
\int f^{2} \log |f| d \mu \leqq c \int\left|\nabla_{\Lambda} f\right|^{2} d \mu+\|f\|_{L^{2}(\mu)}^{2} \log \|f\|_{L^{2}(\mu)}, \quad f \in C_{\Lambda}^{1}(\mathbf{M})
$$

When $c(\mu ; \Lambda)<\infty$, we say that $\mu$ admits a logarithmic Sobolev inequality on $\Lambda$, in which case, $(1.1)$ with $c=c(\mu ; \Lambda)$ is the logarithmic Sobolev constant for $\mu$ on $\Lambda$; and when $\Lambda=\mathbb{Z}^{d}$, we drop all reference to $\Lambda$ in the notation. Thus, $c(\mu)=c\left(\mu ; \mathbb{Z}^{d}\right)$.

Potentials and Gibbs States. The family $\mathscr{J}=\left\{J_{A}: A \in \mathfrak{F}\right\}$ is a twice differentiable, shift-invariant potential with finite range $R \in \mathbb{Z}^{+}$. That is, for each $A \in \mathfrak{F}, J_{A} \in C_{A}^{2}(\mathbf{M})$, $J_{\mathbf{k}+A}=J_{A} \circ \theta^{\mathbf{k}}, \mathbf{k} \in \mathbb{Z}^{d}$, and $J_{A} \equiv 0$ if $\mathbf{0} \in A \nsubseteq[-R, R]^{d}$. Given $\mathscr{J}$, we define the corresponding local specification $\mathfrak{E}=\mathfrak{E}(\mathscr{J})$ so that

$$
\begin{aligned}
{\left[E^{\Lambda} f\right](\xi) } & =\int_{\mathbf{M}} f(\mathbf{y}) E^{\Lambda}(d \mathbf{y} \mid \xi) \\
& =\frac{1}{Z_{\Lambda}(\xi)} \int_{\mathbf{M}} f\left(\mathbf{y}_{\Lambda} \cdot \xi_{\Lambda C}\right) \exp \left[-U^{\Lambda}\left(\mathbf{y}_{\Lambda} \cdot \xi_{\Lambda C}\right)\right] \lambda(d \mathbf{y}),
\end{aligned}
$$

where $\lambda \equiv \lambda^{\mathbb{Z}^{d}}$

$$
U^{\Lambda} \equiv \sum_{\substack{A \in \mathfrak{J} \\ A \cap \Lambda \neq \varnothing}} J_{A}, \quad \text { and } \quad Z_{\Lambda}(\xi) \equiv \int_{\mathbf{M}} \exp \left[-U^{\Lambda}\left(\mathbf{y}_{\Lambda} \cdot \xi_{\Lambda \mathrm{C}}\right)\right] \lambda(d \mathbf{y})
$$

In this connection, it will be convenient for us to have the notation

$$
\langle f\rangle_{\Lambda, \xi} \equiv \int_{\mathbf{M}} f(\mathbf{y}) E^{\Lambda}(d \mathbf{y} \mid \xi) \text { and }\|f\|_{p, \Lambda, \xi} \equiv\left(\int_{\mathbf{M}}|f(\mathbf{y})|^{p} E^{\Lambda}(d \mathbf{y} \mid \xi)\right)^{1 / p} \text {. }
$$

Finally, we say that $\mu \in \mathfrak{M}_{1}(\mathbf{M})$ is a Gibbs state for $\mathfrak{E}(\mathscr{J})$ and write $\mu \in \mathfrak{G}(\mathscr{J})$ if

$$
\left\langle E^{\Lambda} f\right\rangle_{\mu}=\langle f\rangle_{\mu} \text { for all } \Lambda \in \mathfrak{F} \text { and } f \in C_{0}(\mathbf{M}) \text {. }
$$

With this notation in place, we can now state the central result of the present article.

1.2 Theorem. If for each $f \in C_{0}^{2}(\mathbf{M})$,

$$
\sum_{\mathbf{k} \in \mathbb{Z}^{d}}|\mathbf{k}|^{d} \sup _{\substack{\boldsymbol{c} \in \mathfrak{J} \\ \Lambda \ni \mathbf{k}}}\left\|E^{\Lambda \backslash\langle\mathbf{k}\}}\left(f-E^{\Lambda} f\right)\right\|_{u}<\infty
$$

then, for each non-empty $S \subset \mathbb{Z}^{d}$ and $\mathbf{z} \in \mathbf{M}$, there is a unique $E^{S}(\cdot \mid \mathbf{z}) \in \mathfrak{M}_{1}(\mathbf{M})$ with the property that

$$
\int_{\mathbf{M}} f(\mathbf{x}) E^{S}(d \mathbf{x} \mid \mathbf{z})=\int_{\mathbf{M}}\left[E^{\Lambda} f\right]\left(\mathbf{y}_{S} \cdot \mathbf{z}_{S C}\right) E^{S}(d \mathbf{y} \mid \mathbf{z}), \quad S \supset \Lambda \in \mathfrak{F} \quad \text { and } f \in C_{0}(\mathbf{M}) .
$$

In particular, $\mathscr{G}(\mathscr{J})$ contains precisely one element $\mu$; and, for each non-empty $S \subset \mathbb{Z}^{d}$, $\mathbf{z} \in \mathbf{M} \mapsto E^{S}(\cdot \mid \mathbf{z}) \in \mathfrak{M}_{1}(\mathbf{M})$ is a continuous transition probability which is a conditional 
probability distribution of $\mu$ given $\mathscr{F}_{\text {sc. Finally, }}$

$$
\sup _{\substack{\varnothing \neq S \subset \mathbb{Z}^{d} \\ \mathbf{z} \in \mathbf{M}}} c\left(E^{S}(\cdot \mid \mathbf{z}) ; S\right)<\infty
$$

and so $\mu$ itself admits a logarithmic Sobolev inequality on $\mathbb{Z}^{d}$.

Conversely, if

$$
\sup _{\substack{\boldsymbol{\Lambda} \in \mathfrak{F} \\ \mathbf{z} \in \mathbf{M}}} c\left(E^{\Lambda}(\cdot \mid \mathbf{z}) ; \Lambda\right)<\infty,
$$

then there exists an $\varepsilon \in(0, \infty)$ such that, for every $A \in \mathfrak{F}$,

$$
\sup _{\substack{\boldsymbol{A} \in \mathfrak{F} \\ \boldsymbol{\Lambda} \ni \mathbf{k}}}\left\|E^{\boldsymbol{\Lambda} \backslash\{\mathbf{k}\}}\left(f-E^{\boldsymbol{\Lambda}} f\right)\right\|_{\mathbf{u}} \leqq K(A) e^{-\varepsilon|\mathbf{k}-\boldsymbol{A}|}\left\|\nabla_{\boldsymbol{A}} f\right\|_{\mathrm{u}}, \quad f \in C_{\boldsymbol{A}}^{2}(\mathbf{M}),
$$

for some $K(A) \in(0, \infty)$. (Cf. Remark 2.18 below for a slight weakening of the conditions under this converse assertion can be proved.)

1.6 Remark. The first part of Theorem 1.2 is covered in [S\&Z]. In fact, in Theorem 3.8 of that article we showed that the conclusions drawn in the first part of Theorem 1.2 above are guaranteed by conditions which involve only $\Lambda$ 's contained in a sufficiently large cube. In particular (cf. Corollary 3.12 of [S\&Z]) this led to the important conclusion that (1.3) for the potential $\mathscr{J}$ implies (1.4) not only for the local specification $\mathfrak{E}(\mathscr{J})$ but also for all local specifications $\mathfrak{E}(\hat{\mathscr{J}})$ corresponding to differentiable, shift-invariant potentials $\hat{J}$ with range $R$ with the property that

$$
\sum_{F \ni 0}\left\|\nabla_{F}\left(\hat{J}_{F}-J_{F}\right)\right\|_{u} \quad \text { is sufficiently small. }
$$

Thus, it is the converse assertion in Theorem 1.2 on which we will be concentrating in this article. Notice that the estimate in (1.5) is what we called the DobrushinShlosman mixing condition in Remark 3.15 of [S\&Z]. In fact, (1.5) is (cf. (3.11) in [Dob\&S, 1] or IIIc in both [Dob\&S, 2] and [Dob\&S, 3]) one of the many equivalent conditions which, as Dobrushin and Shlosman show, imply complete analytic dependence of the corresponding Gibbs states. Hence, one interpretation of Theorem 1.2 is that it says (1.4) can be appended to Dobrushin and Shlosman's list of equivalent conditions. In this connection, it may be worth pointing out that if one combines Theorem 3.8 of [S\&Z] with Theorem 1.2 here, one obtains an alternative proof that complete analyticity admits a characterization in terms of what Dobrushin and Shlosman call a constructive condition (cf. IIIe in [Dob\&S, 2] and CA in Sects. 4 and 5 of [Dob\&S,3]). Unfortunately, Dobrushin and Shlosman's picture of "a big table with a small duster" (cf. Sect. 5 of [Dob\&S, 3]) does not appear when one uses our method; on the other hand, we feel that the addition of (1.4) to Dobrushin and Shlosman's list provides significant confirmation of Tolstoy's wisdom as interpreted by Dobrushin and Shlosman in the first sentence of [Dob\&S, 3].

1.7 Remark. Readers who are familiar with [Dob\&S, 1-3] may be justifiably concerned about the difference between the settings in which we and they work. Indeed, much of what they did was proved only for finite spin spaces, whereas we have worked exclusively with continuous spin spaces. Moreover, there are several 
places in both their and our analyses where it may not be entirely trivial to extend the argument so that it covers both finite and continuous spins. Nonetheless, we firmly believe that essentially all their results extend to continuous spins and that ours can be proved for finite spins. In fact, the only question about the extension of our results is whether one can carry out the analysis in Sect. 2 of [S\&Z] when one has to replace the classical Leibniz rule by its discrete analogue; that the proof, given below, of $(1.4) \Rightarrow(1.5)$ works (and is in fact easier) in the finite spin case should be clear.

In our proof that (1.4) implies (1.5), we will be borrowing heavily from the ideas introduced in $[\mathrm{H} \& \mathrm{~S}, 1]$ and $[\mathrm{H} \& \mathrm{~S}, 2]$. In particular, this means that we will have to discuss Glauber-type dynamics associated with the potential $\mathscr{J}$ and will be taking advantage of properties of these dynamics which result from our assumption that $\mathscr{J}$ has finite range. Thus, in order to facilitate the presentation later, we will now present an elementary, abstract statement of what will turn out to be the key fact about Glauber dynamics corresponding to finite range potentials.

In the following, $\left\{T_{t}: t>0\right\}$ denotes a Feller continuous, Markov semigroup on $C(\mathbf{M})$, and $\mathbf{A}$ on domain $\mathbf{D}(\mathbf{A})$ is the generator of $\left\{T_{t}: t>0\right\}$. Given a dense subset $\mathfrak{D}$ of $C(\mathbf{M})$, we will say that $\left\{T_{t}: t>0\right\}$ is $\mathfrak{D}$-decomposable if $\mathfrak{D}$ is a $\left\{T_{t}: t>0\right\}$ invariant subset of $\mathbf{D}(\mathbf{A})$ and, for each $\mathbf{k} \in \mathbb{Z}^{d}$, there is an operator $\mathbf{A}_{\mathbf{k}}: \mathfrak{D} \rightarrow C(\mathbf{M})$ with the properties that $\mathbf{A}_{\mathbf{k}} f \equiv 0$ whenever $f \in \mathfrak{D}$ is independent of $x_{\mathbf{k}}$ and, for all $f \in \mathfrak{D}$,

$$
\sum_{\mathbf{k} \in \mathbb{Z}^{d}}\left\|\mathbf{A}_{\mathbf{k}} f\right\|_{\mathbf{u}}<\infty \quad \text { and } \quad \mathbf{A} f=\sum_{\mathbf{k} \in \mathbb{Z}^{d}} \mathbf{A}_{\mathbf{k}} f
$$

in which case $\left\{\mathbf{A}_{\mathbf{k}}: \mathbf{k} \in \mathbb{Z}^{d}\right\}$ is said to be the corresponding $\mathfrak{D}$-decomposition of $\mathbf{A}$. In keeping with our earlier conventions, we will use $\mathfrak{D}_{\Lambda}$ to denote $\mathfrak{D} \cap C_{\Lambda}(\mathbf{M})$.

1.8 Lemma. Let the Feller continuous, Markov semigroup $\left\{T_{t}: t>0\right\}$ with generator $\mathbf{A}$ be $\mathfrak{D}$-decomposable and $\left\{\mathbf{A}_{\mathbf{k}}: \mathbf{k} \in \mathbb{Z}^{d}\right\}$ the corresponding $\mathfrak{D}$-decomposition of $A$. Further, suppose that, for each $\mathbf{k} \in \mathbb{Z}^{d},\|\cdot\|_{\mathbf{k}}$ is a semi-norm on $\mathfrak{D}$ with the property that

$$
\left\|T_{t} f\right\|_{\mathbf{k}} \leqq\|f\|_{\mathbf{k}}+\frac{C}{(2 R+1)^{d}} \sum_{\{\mathbf{j}:|\mathbf{j}-\mathbf{k}| \leqq R\}} \int_{0}^{t}\left\|T_{s} f\right\|_{\mathbf{j}} d s
$$

for some $R \in(0, \infty), C \in(0, \infty)$, and all $f \in \mathfrak{D}, t \in(0, \infty)$, and $\mathbf{k} \in \mathbb{Z}^{d}$. Then, for all $\Lambda \subseteq \mathbb{Z}^{d}$, $N \in \mathbb{N}$, and $f \in \mathfrak{D}_{\Lambda}$,

$$
\sum_{\{\mathbf{k}:|\mathbf{k}-\Lambda| \geqq N R\}}\left\|\mid T_{t} f\right\|_{\mathbf{k}} \leqq e_{N}(C t)\|\| f \|_{\Lambda},
$$

where $\|f\|_{\Lambda}$ stands for $\sum_{\mathbf{k} \in \boldsymbol{\Lambda}}\|f \mid\|_{\mathbf{k}}$ and

$$
e_{0}(s)=e^{s} \quad \text { and } \quad e_{N}(s) \equiv e^{s}-\sum_{n=0}^{N-1} \frac{s^{n}}{n !} \leqq\left(\frac{s e}{N}\right)^{N} e^{s}, \quad N \in \mathbb{Z}^{+} .
$$

Next, assume that there is some $S \subseteq \mathbb{Z}^{d}$ for which $\mathfrak{D}_{S}$ is $\left\{T_{t}: t>0\right\}$-invariant. Also, suppose that $\left\{\hat{T}_{t}: t>0\right\}$, with generator $\hat{A}$, is a second Feller continuous, Markov semigroup which is $\mathfrak{D}$-decomposable, and let $\left\{\hat{\mathbf{A}}_{\mathbf{k}}: \mathbf{k} \in \mathbb{Z}^{d}\right\}$ be the corresponding $\mathfrak{D}$-decomposition of $\hat{A}$. Finally, assume that

$$
\left\|\mathbf{A}_{\mathbf{k}} f-\hat{\mathbf{A}}_{\mathbf{k}} f\right\|_{\mathbf{u}} \leqq C\|f\|_{\mathbf{k}}, \quad \mathbf{k} \in S \text { and } f \in \mathfrak{D}_{S}
$$


and that, for some $N \in \mathbb{N}$ and $\Lambda \subseteq S$,

$$
\widehat{\mathbf{A}}_{\mathbf{k}}=\mathbf{A}_{\mathbf{k}} \text { for all } \mathbf{k} \in S \text { with }|\mathbf{k}-\Lambda|<N R \text {. }
$$

Then,

$$
\left\|T_{t} f-\hat{T}_{t} f\right\|_{\mathbf{u}} \leqq e_{N+1}(C t)\|f\|_{\Lambda}, \quad f \in \mathfrak{D}_{\Lambda} .
$$

Hence, if, in addition, there exist $\mu, \hat{\mu} \in \mathfrak{M}_{1}(\mathbf{M})$ with the property that

$$
\rho_{f}(t) \equiv\left\|T_{t} f-\langle f\rangle_{\mu}\right\|_{\mathbf{u}} \vee\left\|\hat{T}_{t} f-\langle f\rangle_{\hat{\mu}}\right\|_{\mathbf{u}} \searrow 0 \text { as } t>\infty
$$

for each $f \in \mathfrak{D}_{\Lambda}$, then

$$
\left|\langle f\rangle_{\mu}-\langle f\rangle_{\hat{\mu}}\right| \leqq R_{f}(N) \equiv \inf \left\{\rho_{f}(t)+e_{N+1}(C t)\|f\|_{\Lambda}: t \in(0, \infty)\right\} .
$$

In particular, if there is an $\alpha \in(0, \infty)$ and a $C(\Lambda) \in(0, \infty)$ for which

$$
\left\|T_{t} f-\langle f\rangle_{\mu}\right\|_{\mathbf{u}} \vee\left\|\hat{T}_{t} f-\langle f\rangle_{\hat{\mu}}\right\|_{\mathbf{u}} \leqq C(\Lambda)\|f\|_{\Lambda} e^{-\alpha t}, \quad t \in(0, \infty) \text { and } f \in \mathfrak{D}_{\Lambda} \text {, }
$$

then there is a $\beta=\beta(\alpha, C) \in(0, \infty)$ such that

$$
\left|\langle f\rangle_{\mu}-\langle f\rangle_{\hat{\mu}}\right| \leqq 2 C(\Lambda)\|f\|_{\Lambda} e^{-\beta N}, \quad f \in \mathfrak{D}_{\Lambda} .
$$

Proof. Define $a_{N}(t)$ to be the left-hand side of (1.10) and observe that, because of (1.9),

$$
a_{0}(t) \leqq a_{0}(0) e^{C t} \quad \text { and } \quad a_{N}(t) \leqq \int_{0}^{t} a_{N-1}(s) d s \text { for } \quad N \in \mathbb{Z}^{+}
$$

Hence, (1.10) follows immediately by induction with respect to $N \in \mathbb{N}$.

Turning to (1.13), set $f_{t}=T_{t} f$ and observe that, because of (1.12) and the fact that $f_{t} \in \mathfrak{D}_{S}$ for all $t \in[0, \infty)$,

$$
\frac{d}{d s} \hat{T}_{t-s} f_{s}=\hat{T}_{t-s}{ }^{\circ}(\mathbf{A}-\hat{\mathbf{A}}) f_{s}=\sum_{\{\mathbf{k} \in S:|\mathbf{k}-\Lambda| \geqq N R\}} \hat{T}_{t-s}{ }^{\circ}\left(\mathbf{A}_{\mathbf{k}}-\hat{\mathbf{A}}_{\mathbf{k}}\right) f_{t}
$$

for $s \in(0, t)$. Hence

$$
\left\|T_{t} f-\hat{T}_{t} f\right\|_{\mathbf{u}} \leqq \int_{0}^{t}\|h(s)\|_{\mathbf{u}} d s, \quad \text { where } \quad h(s) \equiv \sum_{\{\mathbf{k} \in S:|\mathbf{k}-\Lambda| \geqq N R\}}\left(\mathbf{A}_{\mathbf{k}}-\hat{\mathbf{A}}_{\mathbf{k}}\right) f_{t} ;
$$

and therefore, by (1.10) and (1.11),

$$
\left\|T_{t} f-\hat{T}_{t} f\right\|_{\mathrm{u}} \leqq C\|f\|_{\Lambda} \int_{0}^{t} e_{N}(C s) d s=e_{N+1}(C t)\|f\|_{\Lambda}
$$

Finally, (1.14) is an essentially trivial consequence of (1.13); and to prove (1.16) when $(1.15)$ holds, simply note that by taking $t=\frac{(N+1) \gamma}{C}$, where $\gamma \in(0, \infty)$ solves

$$
\left(1+\frac{\alpha}{C}\right) \gamma+\log \gamma+1=0
$$

one can dominate $R_{f}(N)$ in (1.14) by

$$
2 C(\Lambda)\|f \mid\|_{\Lambda} \exp \left[-\frac{\alpha \gamma}{C}(N+1)\right] \text {. }
$$




\section{Continuous Glauber Dynamics}

In this section we will prove the second (i.e., the converse) assertion in Theorem 1.2; and to this end, for each $\Lambda \in \mathfrak{F}$ and $\xi \in \mathbf{M}$, we introduce the diffusion semigroup $\left\{P_{t}^{\Lambda, \xi}: t>0\right\}$ for which the measures $E^{\Lambda}(\cdot \mid \xi)$ is the equilibrium state and

$$
\mathscr{E}^{\Lambda, \xi}(\varphi, \varphi) \equiv \sum_{\mathbf{k} \in \boldsymbol{\Lambda}} \int\left|\nabla_{\mathbf{k}} \varphi\right|^{2} E^{\Lambda}(d \mathbf{y} \mid \boldsymbol{\xi})
$$

is the Dirichlet form. That is, $\left\{P_{t, \xi}^{\Lambda, \xi}: t>0\right\}$ is the unique Feller continuous, Markov semigroup whose generator $\mathbf{A}^{\Lambda, \xi}$ restricted to $C_{0}^{2}(\mathbf{M})$ is given by

$$
\left[\mathbf{A}^{\Lambda, \xi} f\right](\mathbf{x})=\sum_{\mathbf{k} \in \Lambda}\left[e^{U^{\Lambda, \xi}} \operatorname{div}_{\mathbf{k}}\left(e^{-U^{\Lambda, \xi}} \nabla_{\mathbf{k}} f\right)\right](\mathbf{x}),
$$

where $U^{\Lambda, \xi}(\mathbf{x}) \equiv U^{\Lambda}\left(\mathbf{x}_{\Lambda} \cdot \xi_{\Lambda C}\right)$. Taking advantage of the fact that $\left\{P_{t}^{\Lambda, \xi}: t>0\right\}$ acts trivially on all but a finite number of coordinates, standard facts about finite dimensional diffusions apply. In particular, we have the following.

2.3 Lemma. Each of the spaces $C_{\Lambda}(\mathbf{M}), C_{0}^{1}(\mathbf{M})$, and $C_{0}^{2}(\mathbf{M})$ is invariant under $\left\{P_{t}^{\Lambda, \xi}: t>0\right\}$. In addition, there is a constant $K \in[1, \infty)$, which is independent of both $\Lambda$ and $\xi$, such that

$$
\left\|P_{1}^{\Lambda, \xi} f\right\|_{\mathbf{u}} \leqq K^{|\Lambda|}\|f\|_{1, \Lambda, \xi}, \quad f \in C(\mathbf{M}) .
$$

Proof. The invariance properties of $\left\{P_{t}^{\Lambda, \xi}: t>0\right\}$ are completely standard facts about finite dimensional heat flows. To prove (2.4), let $f$ be a non-negative element of $C^{2}(\mathbf{M})$, set

$$
w(t, \mathbf{x})=\exp \left[-\frac{U^{\Lambda, \xi}(\mathbf{x})}{2}\right]\left[P_{t}^{\Lambda, \xi} f\right](\mathbf{x}) . \text { for } \quad(t, \mathbf{x}) \in[0, \infty) \times \mathbf{M},
$$

and note that

$$
\frac{\partial w}{\partial t}(t, \mathbf{x})=\sum_{\mathbf{k} \in \Lambda}\left[\Delta_{\mathbf{k}} w\right](t, \mathbf{x})-V^{\Lambda, \xi}(\mathbf{x}) w(t, \mathbf{x}),
$$

where $\Delta_{\mathbf{k}} \varphi \equiv \operatorname{div}_{\mathbf{k}}\left(\nabla_{\mathbf{k}} \varphi\right)$ for $\mathbf{k} \in \mathbb{Z}^{d}$ and $\varphi \in C^{2}(\mathbf{M})$, and

$$
V^{\Lambda, \xi} \equiv \sum_{\mathbf{k} \in \Lambda} \exp \left[\frac{U^{\Lambda, \xi}}{2}\right] \Delta_{\mathbf{k}}\left(\exp \left[-\frac{U^{\Lambda, \xi}}{2}\right]\right)
$$

Since, by any one of many (e.g., the Feynman-Kac formula) standard arguments,

$$
|w(t, \mathbf{x})| \leqq \exp \left[t\left\|V^{\Lambda, \xi}\right\|_{\mathbf{u}}\right] w_{0}(t, \mathbf{x}),
$$

where

$$
\frac{\partial w_{0}}{\partial t}(t, \mathbf{x})=\sum_{\mathbf{k} \in A}\left[\Delta_{\mathbf{k}} w_{0}\right](t, \mathbf{x}) \quad \text { with } \quad w_{0}(0, \cdot)=f,
$$

we now know that

$$
\left[P_{1}^{\Lambda, \xi} f\right](\mathbf{x}) \leqq \exp \left[\frac{U^{\Lambda, \xi}(\mathbf{x})}{2}+\left\|V^{\Lambda, \xi}\right\|_{\mathbf{u}}\right] w_{0}(1, \mathbf{x}) .
$$


Finally, if $p_{0}(t, x, y)$ is the heat kernel for $M$, then

$$
\begin{aligned}
\exp \left[\frac{U^{\Lambda, \xi}(\mathbf{x})}{2}\right] w_{0}(1, \mathbf{x}) & =\int_{\mathbf{M}}\left(\prod_{\mathbf{k} \in \Lambda} p_{0}\left(1, x_{\mathbf{k}}, y_{\mathbf{k}}\right)\right) \exp \left[\frac{U^{\Lambda, \xi}(\mathbf{x})-U^{\Lambda, \xi}(\mathbf{y})}{2}\right] f(\mathbf{y}) \lambda(d \mathbf{y}) \\
& \leqq e^{2 \operatorname{osc}\left(U^{\Lambda, \xi}\right)} C^{|\Lambda|} \int_{\mathbf{M}} f(\mathbf{y}) E^{\Lambda}(d \mathbf{y} \mid \xi)
\end{aligned}
$$

where $C \in(0, \infty)$ is the maximum value of $p_{0}(1, x, y)$ and

$$
\operatorname{osc}\left(U^{\Lambda, \xi}\right) \equiv \sup _{\mathbf{x}, \mathbf{y} \in \mathbf{M}}\left|U^{\Lambda, \xi}(\mathbf{y})-U^{\Lambda, \xi}(\mathbf{x})\right| \leqq \kappa|\Lambda|
$$

for some $\kappa \in(0, \infty)$. Hence, since it is clear that $\left\|V^{\Lambda, \xi}\right\|_{\mathbf{u}}$ is dominated by a constant times $|\Lambda|$, the derivation of $(2.4)$ is complete.

Combining the first part of the preceding with the presentation of $\mathbf{A}^{\Lambda, \xi}$ in (2.2), we see that each $\left\{P_{t}^{\Lambda, \xi}: t>0\right\}$ is $C_{0}^{2}(\mathbf{M})$-decomposable. Thus, the results of Lemma 1.8 will be applicable once we have the following.

\subsection{Lemma. Define}

$$
\|f\|_{\mathbf{k}}=\left\|\left|\nabla_{\mathbf{k}} f\right|\right\|_{\mathbf{u}}, \quad f \in C^{1}(\mathbf{M}) \text { and } \mathbf{k} \in \mathbb{Z}^{d} .
$$

Then a constant $C \in(0, \infty)$ can be chosen so that, for all $\Lambda \in \mathfrak{F}, \boldsymbol{\xi} \in \mathbf{M}, \mathbf{k} \in \mathbb{Z}^{d}$, and $f \in C_{0}^{2}(\mathbf{M})$ :

$$
\left\|P_{t}^{\Lambda, \xi} f\right\|_{\mathbf{k}} \leqq\|f\|_{\mathbf{k}}+\frac{C}{(2 R+1)^{d}} \sum_{\{\mathbf{j} \in \Lambda:|\mathbf{j}-\mathbf{k}| \leqq R\}} \int_{0}^{t}\left\|P_{s}^{\Lambda, \xi} f\right\|_{\mathbf{j}} d s, \quad t \in(0, \infty),
$$

and, for all $\Lambda, \hat{\Lambda} \in \mathfrak{F}$ and $\mathbf{k} \in \Lambda \cap \hat{\Lambda}$,

$$
\left\|\mathbf{A}_{\mathbf{k}}^{\Lambda, \xi} f-\mathbf{A}_{\mathbf{k}}^{\hat{\Lambda}, \xi} f\right\|_{\mathbf{u}} \leqq C\|f\|_{\mathbf{k}}, \quad f \in C_{0}^{2}(\mathbf{M}) .
$$

In particular, for all $\Lambda, \hat{\Lambda} \in \mathfrak{F}$ with $\Lambda \subseteq \hat{\Lambda}$, all $A \subseteq \Lambda$, and all $\boldsymbol{\xi}, \hat{\xi} \in \mathbf{M}$ satisfying

$$
(\hat{\Lambda} \backslash \Lambda) \cup\left\{\mathbf{k} \notin \Lambda: \xi_{\mathbf{k}}^{\prime} \neq \xi_{\mathbf{k}}\right\} \subseteq\left\{\mathbf{k} \in \mathbb{Z}^{d}:|\mathbf{k}-A| \geqq N R\right\},
$$

one has (cf. (1.10)),

$$
\left\|P_{t}^{\Lambda, \xi} f-P_{t}^{\hat{\Lambda}, \xi^{\prime}} f\right\|_{\mathbf{u}} \leqq e_{N}(C t) \sum_{\mathbf{k} \in A}\|f\|_{\mathbf{k}}, \quad f \in C^{2}(\mathbf{M}) .
$$

Proof. Since (2.7) is obvious and, given (2.6) and (2.7), (2.8) is an easy application of Lemma 1.8, we will restrict our attention to (2.6). To this end, we first introduce $H_{\mathbf{j}, \mathbf{k}}(\psi)$ for $\psi \in C^{2}(\mathbf{M})$ and $\mathbf{j}, \mathbf{k} \in \mathbb{Z}^{d}$ to be the $(\mathbf{j}, \mathbf{k})^{\text {th }}$ block of the Hessian tensor of $\psi$. It is then an elementary application of Bochner's formula to see that

$$
\begin{aligned}
2\left(\nabla_{\mathbf{k}} \psi \mid \nabla_{\mathbf{k}} \mathbf{A}_{\mathbf{j}}^{\Lambda, \xi} \psi\right)= & \mathbf{A}_{\mathbf{j}}^{\Lambda, \xi}\left(\left|\nabla_{\mathbf{k}} \psi\right|^{2}\right)-\delta_{\mathbf{j}, \mathbf{k}} \operatorname{Ric}\left(\nabla_{\mathbf{j}} \psi, \nabla_{\mathbf{k}} \psi\right) \\
& -H_{\mathbf{j}, \mathbf{k}}\left(U^{\Lambda, \xi}\right)\left(\nabla_{\mathbf{j}} \psi, \nabla_{\mathbf{k}} \psi\right)-\left\|H_{\mathbf{j}, \mathbf{k}}(\psi)\right\|_{\text {H.S. }}^{2},
\end{aligned}
$$

where Ric denotes the Ricci curvature tensor for $M$ and the norm in the last expression is the Hilbert-Schmidt norm. In particular, if $f \in C_{0}^{2}(\mathbf{M})$ and $f_{t} \equiv P_{t}^{\Lambda, \xi} f$, then

$$
\frac{d}{d t}\left|\nabla_{\mathbf{k}} f_{t}\right|^{2} \leqq \mathbf{A}^{\Lambda, \xi}\left(\left|\nabla_{\mathbf{k}} f_{t}\right|^{2}\right)-\operatorname{Ric}\left(\nabla_{\mathbf{k}} f_{t}, \nabla_{\mathbf{k}} f_{t}\right)-\sum_{\mathbf{j} \in \Lambda} H_{\mathbf{j}, \mathbf{k}}\left(U^{\Lambda, \xi}\right)\left(\nabla_{\mathbf{j}} f_{t}, \nabla_{\mathbf{k}} f_{t}\right) .
$$


Thus, by considering $s \in[0, t] \mapsto P_{t-s}^{\Lambda, \xi}\left(\left|\nabla_{\mathbf{k}} f_{s}\right|^{2}\right) \in \mathbb{R}$, we see first that there is a $C \in(0, \infty)$ for which

$$
\|\| f_{t}\left\|_{\mathbf{k}}^{2} \leqq\right\| f\left\|_{\mathbf{k}}^{2}+\frac{C}{(2 R+1)^{d}} \sum_{\{\mathbf{j} \in \Lambda:|\mathrm{j}-\mathbf{k}| \leqq R\}} \int_{0}^{t}\right\| f_{s}\left\|_{\mathbf{j}}\right\| f_{s} \|_{\mathbf{k}} d s,
$$

and thence that (2.6) holds with the same choice of $C$.

So far, nothing which we have done in this section makes any use of the condition in (1.4). Indeed, we use it for the first time when we attempt to check (1.15) holds uniformly for the semigroups $\left\{P_{t}^{\Lambda, \xi}: t>0\right\}$. To be precise, we have the following.

2.9 Lemma. Set (cf. (2.1))

$$
\gamma(\Lambda, \xi)=\inf \left\{\frac{\mathscr{E}^{\Lambda, \xi}(\varphi, \varphi)}{\left[E^{\Lambda} \varphi^{2}\right](\xi)}: \varphi \in C_{0}^{2}(\mathbf{M}) \text { with }\left[E^{\Lambda} \varphi\right](\xi)=0\right\}
$$

for all $\Lambda \in \mathfrak{F}$ and $\xi \in \mathbf{M}$. Then,

$$
\gamma(\Lambda, \xi) \geqq \frac{1}{c\left(E^{\Lambda}(\cdot \mid \xi) ; \Lambda\right)} .
$$

Moreover, if (1.4) holds, then, for every $\theta \in(0,1)$ and $A \in \mathfrak{F}$, there is a $K_{\theta}(A) \in(0, \infty)$ such that

$$
\left\|P_{t}^{\Lambda, \xi} f-\left[E^{\Lambda} f\right](\xi)\right\|_{\mathbf{u}} \leqq K_{\theta}(A) e^{-\theta \gamma(\Lambda, \xi) t}\|f\|_{A}, \quad f \in C_{A}^{2}(\mathbf{M}) \quad \text { and } \quad(t, \xi) \in(0, \infty) \times \mathbf{M} \text {. }
$$

In particular, if

$$
\frac{1}{\alpha} \equiv 2 \sup \left\{c\left(E^{\Lambda}(\cdot \mid \xi) ; \Lambda\right): \Lambda \in \mathfrak{F} \text { and } \boldsymbol{\xi} \in \mathbf{M}\right\}
$$

then

$$
\left\|P_{t}^{\Lambda, \xi} f-\left[E^{\Lambda} f\right](\xi)\right\|_{\mathbf{u}} \leqq K(A) e^{-\alpha t}\|f\|_{A}, \quad f \in C_{A}^{2}(\mathbf{M}) \quad \text { and } \quad(t, \xi) \in(0, \infty) \times \mathbf{M},
$$

with $K(A)=K_{1 / 2}(A)$.

Proof. Equation (2.11) is (cf. Corollary 6.1.17 in [D\&S]) a simple restatement of a remark by B. Simon (cf. Corollary 6.1 .7 in [D\&S]). Thus, from now on we will assume that (1.4) holds and will be taking $\alpha$ accordingly, as in the last part of the statement.

By L. Gross's fundamental result in $[\mathrm{G}, 1]$ (alternatively, again see Corollary 6.1.17 in [D\&S]), we know that, for all $t \in(0, \infty), \Lambda \in \mathfrak{F}$, and $\varphi \in C(\mathbf{M})$ :

$$
\left\|P_{t}^{\Lambda, \xi} \varphi\right\|_{q(t), \Lambda, \xi} \leqq\|\varphi\|_{2, \Lambda, \xi}, \text { with } q(t)=1+e^{2 \alpha t} .
$$

At the same time, by elementary spectral theory,

$$
\left\|P_{t}^{\Lambda, \xi} \varphi-\left[E^{\Lambda} \varphi\right](\xi)\right\|_{2, \Lambda, \xi} \leqq e^{-\gamma(\Lambda, \xi) t}\left\|\varphi-\left[E^{\Lambda} \varphi\right](\xi)\right\|_{2, \Lambda, \xi}, \quad \varphi \in C(\mathbf{M}),
$$

for all $\Lambda \in \mathfrak{F}$ and $(t, \xi) \in(0, \infty) \times \mathbf{M}$.

Now let $A \in \mathfrak{F}$ and $\theta \in(0,1)$ be given, and, for $\mathfrak{F} \ni \Lambda \supseteqq A$, define

$$
t \in(0, \infty) \mapsto \Lambda_{A}(t)=\{\mathbf{k} \in \Lambda:|\mathbf{k}-A| \leqq((\sigma+C) t+1) R\} \in \mathfrak{F}
$$


where $\sigma=e^{2}(C \vee G)$ with $C$ the constant appearing in (2.8) and

$$
G \equiv \sup \{\gamma(\Lambda, \xi): \Lambda \in \mathfrak{F} \text { and } \boldsymbol{\xi} \in \mathbf{M}\} \text {. }
$$

(Trivial considerations show that $G<\infty$.) Then, by $(2.8)$, we know that

$$
\left\|P_{t}^{\Lambda, \xi} f-P_{t}^{\Lambda_{A}(t), \xi} f\right\|_{\mathrm{u}} \leqq e^{-\sigma t}\|f\|_{A}, \quad f \in C_{A}^{2}(\mathbf{M})
$$

and so

$$
\| P_{t}^{\Lambda, \xi_{f}}-\left[E^{\Lambda_{A}(t), \xi_{f}} f(\xi)\left\|_{\mathrm{u}} \leqq e^{-\sigma t}\right\| f\left\|_{A}+\right\| P_{t}^{\Lambda_{A}(t), \xi_{f}} f-\left[E^{\Lambda_{A}(t), \xi_{j}} f\right](\xi) \|_{\mathrm{u}} .\right.
$$

At the same time, by (2.4) and standard interpolation theory,

$$
\begin{array}{r}
\left\|P_{1}^{\Lambda, \xi} \varphi-\left[E^{\Lambda, \xi} \varphi\right](\xi)\right\|_{\mathbf{u}} \leqq \\
K^{|\Lambda| / q}\left\|\varphi-\left[E^{\Lambda, \xi} \varphi\right](\xi)\right\|_{q, \Lambda, \xi} \\
\varphi \in C(\mathbf{M}), \quad \Lambda \in \mathfrak{F}, \quad \text { and } \xi \in \mathbf{M}
\end{array}
$$

for every $q \in(1, \infty)$. In particular, if $q_{\theta}(t) \equiv q((1-\theta)(t-1))$ for $t \in[1, \infty)$, then $(2.14)$ and (2.15) show that

$$
\begin{aligned}
& \| P_{t}^{\Lambda_{A}(t), \xi} f-\left[E^{\Lambda_{A}(t), \xi} f\right](\xi) \|_{\mathrm{u}} \\
& \leqq K^{\left|\Lambda_{A}(t)\right| / q_{\theta}(t)}\left\|P_{\theta(t-1)}^{\Lambda_{A}(t), \xi} f-\left[E^{\Lambda_{A}(t)} f\right](\xi)\right\|_{2, \Lambda_{A}(t), \xi} \\
& \leqq K^{\left|\Lambda_{A}(t)\right| / q_{\theta}(t)} e^{-\theta \gamma\left(\Lambda_{A}(t), \xi\right)(t-1)}\left\|f-\left[E^{\Lambda_{A}(t)} f\right](\xi)\right\|_{2, \Lambda_{A}(t), \xi} \\
& \leqq \\
& \quad \operatorname{diam}(M) K^{\left|\Lambda_{A}(t)\right| / q_{\theta}(t)} e^{-\theta \gamma\left(\Lambda_{A}(t), \xi\right) t}\|f\|_{A}, \quad t \in[1, \infty) \text { and } f \in C_{A}^{2}(\mathbf{M}) .
\end{aligned}
$$

Finally, since $t \in[1, \infty) \mapsto \frac{\left|\Lambda_{A}(t)\right|}{q_{\theta}(t)} \in[0, \infty)$ is bounded and $G \geqq \gamma\left(\Lambda_{A}(t), \xi\right) \geqq \gamma(\Lambda, \xi)$, the preceding together with $(2.16)$ makes it clear how $K_{\theta}(A)$ can be chosen so that (2.12) holds.

Proof of Theorem 1.2. As we said following its statement, the first half of Theorem 1.2 was proved in Corollary 3.18 of [S\&Z]. Thus, all that we have to do here is prove that (1.4) implies (1.5). But, by combining Lemmas 2.5 and 2.9 with the last part of Lemma 1.8, we know see that there is a $\beta \in(0, \infty)$ such that, for every $A \in \mathfrak{F}$,

$$
\begin{aligned}
& \left\|E^{\Lambda \backslash\{\mathbf{k}\}} f-E^{\Lambda} f\right\|_{\mathbf{u}} \leqq(K(A)+1) e^{-\beta N} \mid\|f\|_{A}, \\
& f \in C_{A}^{2}(\mathbf{M}) \text { and } \mathbf{k} \in \Lambda \text { with }|\mathbf{k}-A| \geqq N R,
\end{aligned}
$$

where $K(A)$ is the quantity in (2.13); and, obviously, after adjusting $K(A)$, this leads to (1.5) with $\varepsilon=\frac{\beta}{R}$.

2.17 Remark. As the preceding line of reasoning makes clear, we could have added the existence of an $\alpha \in(0, \infty)$ and $\{K(A): A \in \mathfrak{F}\} \subseteq[0, \infty)$ for which (2.13) holds to the list of conditions which are equivalent to (1.4). Moreover, with essentially no change in the argument, one can prove (2.12) as soon as one knows that

$$
\varlimsup_{t \rightarrow \infty}\left(\frac{\log t}{t}\right) \sup \left\{c\left(E^{\Lambda}(\cdot \mid \xi)\right): \varnothing \neq \Lambda \subseteq[-t, t]^{d} \text { and } \xi \in \mathbf{M}\right\}=0 .
$$

Hence (just as in the proof given of (2.13)), (2.18) together with

$$
\inf \{\gamma(\Lambda, \xi): \Lambda \in \mathfrak{F} \text { and } \boldsymbol{\xi} \in \mathbf{M}\}>0
$$


implies first (2.13) for some $\alpha \in(0, \infty)$ and then (1.5) for some $\beta \in(0, \infty)$. In particular, this means that when (2.19) is known to hold, (2.18) is equivalent to the apparently stronger (1.4).

\section{Some Dividends}

Although we have now reached our major objective, namely, the proof of the equivalence contained in Theorem 1.2, there are still a couple of additional observations which can be extracted from what we have done. The first of these is the remark that we are now in a position to extend the reasoning in [H\&S, 2] to the present setting and thereby show that (1.4) implies a sharp exponential rate at which the Glauber dynamics converges to equilibrium. Thus, let $\left\{P_{t}: t>0\right\}$ be the Feller continuous, Markov semigroup for which the Gibbs state $\mu$ is the equilibrium distribution and

$$
\mathscr{E}(\varphi, \varphi) \equiv \sum_{\mathbf{k} \in \mathbb{Z}^{d}} \int\left|\nabla_{\mathbf{k}} \varphi\right|^{2} d \mu
$$

is the Dirichlet form. Alternatively, $\left\{P_{t}: t>0\right\}$ can be described as the unique Feller continuous, Markov semigroup whose generator $\mathbf{A}$ has the property that, for each $A \in \mathfrak{F}$,

$$
\mathbf{A} f=\mathbf{A}^{\Lambda, \xi} f, \quad f \in C_{A}^{2}(\mathbf{M}), \quad \mathfrak{F} \ni \Lambda \supseteqq A \cup \partial_{R} A, \quad \text { and } \quad \xi \in \mathbf{M} .
$$

In particular, from this latter description, it is a trivial matter to see that

$$
\lim _{\Lambda>\mathbb{Z}^{d}(t, \xi) \in[0, T] \times \mathbf{M}} \sup _{t}\left\|P_{t}^{\Lambda, \xi} f-P_{t} f\right\|_{\mathbf{u}}=0 \text { for all } T \in(0, \infty) \text { and } f \in C(\mathbf{M}) \text {; }
$$

and, as we are about to see, (3.1) together with (2.12) yield a very strong statement about the way in which $P_{t}$ tends to equilibrium.

3.2 Theorem. Assume that (1.4) holds, let $\mu$ denote the unique element of $(\mathfrak{5}(\mathscr{J})$, and define

$$
\gamma=\inf \left\{\frac{\mathscr{E}(\varphi, \varphi)}{\|\varphi\|_{L^{2}(\mu)}^{2}}: \varphi \in C_{0}^{2}(\mathbf{M}) \text { with }\langle\varphi\rangle_{\mu}=0\right\}
$$

Then $\frac{1}{\gamma}$ is dominated by the logarithmic Sobolev constant $c(\mu)$,

$$
e^{-\gamma t}=\sup \left\{\left\|P_{t} \varphi-\langle\varphi\rangle_{\mu}\right\|_{L^{2}(\mu)}:\|\varphi\|_{L^{2}(\mu)} \leqq 1\right\} \text { for all } t \in(0, \infty)
$$

and, for each $\theta \in(0,1)$ and $A \in \mathfrak{F}$,

$$
\left\|P_{t} f-\langle f\rangle_{\mu}\right\|_{\mathbf{u}} \leqq K_{\theta}(A) e^{-\theta \gamma t}\|f\|_{A} \text { for all } t \in(0, \infty) \text { and } f \in C_{A}^{2}(\mathbf{M}) \text {, }
$$

where $K_{\theta}(A) \in(0, \infty)$ is the same as it was in (2.12). In particular,

$$
\sup _{\boldsymbol{A} \in \mathfrak{F}} \lim _{t \rightarrow \infty} \frac{1}{t} \log \left[\sup \left\{\left\|P_{t} f-\langle f\rangle_{\mu}\right\|_{\mathrm{u}}: f \in C_{\boldsymbol{A}}^{2}(\mathbf{M}) \quad \text { and } \quad\|f\|_{A} \leqq 1\right\}\right]=-\gamma .
$$

Proof. The domination of $\frac{1}{\gamma}$ by $c(\mu)$ is another application of the remark by B. Simon 
alluded to above. In addition, (3.3) is a simple application of spectral theory, and, obviously, (3.5) is an immediate consequence of (3.3) combined with (3.4). Hence, we need only prove (3.4). To this end, set $\Lambda_{n}=[-n, n]^{d}$, choose and fix some $\xi \in \mathbf{M}$, and set $\mu_{n}=E^{\Lambda_{n}, \xi}(\cdot \mid \xi)$ and (cf. (2.10)) $\gamma_{n}=\gamma\left(\Lambda_{n}, \xi\right)$. Because $\mu$ is the only element of $\mathfrak{G}(\mathscr{J})$, it is easy to check that $\mu_{n} \Rightarrow \mu$ and therefore that

$$
\begin{aligned}
\left\|\varphi-\langle\varphi\rangle_{\mu}\right\|_{L^{2}(\mu)}^{2} & =\lim _{n \rightarrow \infty}\left\|\varphi-\langle\varphi\rangle_{\mu_{n}}\right\|_{L^{2}\left(\mu_{n}\right)}^{2} \\
& \leqq \lim _{n \rightarrow \infty} \gamma_{n}\left\langle\left|\nabla_{\Lambda_{n}} \varphi\right|^{2}\right\rangle_{\mu_{n}}=\varliminf_{n \rightarrow \infty}^{\lim } \gamma_{n} \mathscr{E}(\varphi, \varphi), \quad \varphi \in C_{0}^{2}(\mathbf{M}) .
\end{aligned}
$$

Hence, $\gamma \leqq \lim _{n \rightarrow \infty} \gamma_{n}$. At the same time, by (2.12),(2.12), and an elementary application of the triangle inequality,

$$
\left\|P_{t} f-\langle f\rangle_{\mu}\right\|_{\mathrm{u}} \leqq K_{\theta}(A) \underline{\lim _{n \rightarrow \infty}} e^{-\theta \gamma_{n} t}\|f\|_{A} \quad \text { for } t \in(0, \infty) \text { and } f \in C_{A}^{2}(\mathbf{M})
$$

and so (3.4) is now proved.

We next turn to a variation on a question which was discussed first in $[\mathrm{H}]$ and then again in [A\& $\mathrm{H}]$. Namely, in the context of attractive Ising models with spin space $\{-1,1\}$, Holley showed that the rate at which the Glauber dynamics for the whole system takes local functions to equilibrium is necessarily exponential if it is strictly faster than $t^{-d}$. The proofs (both the one in [H] as well as the simplified one in $[\mathrm{A} \& \mathrm{H}]$ ) of this interesting result rely heavily on the hypothesis that the model is attractive and are therefore too delicate to be entirely replaced by the sort of reasoning which we have been using here. Nonetheless, if one is willing to replace Holley's hypothesis about the whole system with a considerably stronger, uniform one about the Glauber dynamics on finite boxes, then one arrives at the following.

3.6 Theorem. Assume that $\mathfrak{G}(\mathscr{J})=\{\mu\}$, and suppose that for each $f \in C_{0}^{2}(\mathbf{M})$,

$$
\int_{1}^{\infty} t^{2 d-1} \sup \left\{\left\|P_{t}^{\Lambda . \xi} f-\langle f\rangle_{\Lambda . \xi}\right\|_{\mathbf{u}}: \Lambda \in \mathfrak{F} \text { and } \xi \in \mathbf{M}\right\} d t<\infty .
$$

Then (1.3) holds, and so the conclusions of both Theorems 1.2 and 3.2 apply. In particular, (3.7) implies (3.4).

Proof. By plugging (3.7) and (2.8) into (1.14), we see that (1.3) holds for each $f \in C_{0}^{2}(\mathbf{M})$.

An important benefit to having a logarithmic Sobolev inequality is that it allows one to develop the large deviation theory for the empirical distribution of the diffusion determined by the semigroup $\left\{P_{t}: t>0\right\}$. To be more precise, standard Markov process considerations lead to the existence of a continuous mapping

$$
\mathbf{x} \in \mathbf{M} \mapsto \mathbf{P}_{\mathbf{x}} \in \mathfrak{M}_{1}(C([0, \infty) ; \mathbf{M}))
$$

with the property that, for every $n \in \mathbb{Z}^{+}, 0<t_{1}<\cdots<t_{n}$, and $F \in C\left(\mathbf{M}^{n}\right)$,

$$
\begin{aligned}
& \quad \int_{C([0 . \infty) ; \mathbf{M})} F\left(\psi\left(t_{1}\right), \ldots, \psi_{n}\left(t_{n}\right)\right) \mathbf{P}_{\mathbf{x}}(d \psi) \\
& \quad \leqq \int_{\mathbf{M}} \cdots \int_{\mathbf{M}} F\left(\mathbf{y}_{1}, \ldots, \mathbf{y}_{n}\right) P\left(t_{1}, \mathbf{x}, d \mathbf{y}_{1}\right) \cdots P\left(t_{n}-t_{n-1}, \mathbf{y}_{n-1}, d \mathbf{y}_{n}\right),
\end{aligned}
$$


where $\mathbf{x} \in \mathbf{M} \mapsto P(t, \mathbf{x}, \cdot) \in \mathfrak{M}_{1}(\mathbf{M})$ is the transition probability for the operator $P_{t}$. Alternatively, if $\mathscr{B}_{t}$ denotes the $\sigma$-algebra over $C([0, \infty)$; M) generated by $\psi \mapsto \psi(s)$ as $s$ runs over $[0, t]$, then, for each $\mathbf{x} \in \mathbf{M}, \mathbf{P}_{\mathbf{x}}$ can be characterized as the unique probability measure on $C([0, \infty) ; \mathbf{M})$ with the property that, for every $f \in C_{0}^{2}(\mathbf{M})$,

$$
\left(f(\psi(t))-f(\mathbf{x})-\int_{0}^{t}[\mathbf{A} f](\psi(s)) d s, \mathscr{B}_{t}, \mathbf{P}_{x}\right) \text { is a mean-zero martingale. }
$$

Now suppose that $\mu \in \mathfrak{G}(\mathscr{J})$, and set $\mathbf{P}_{\mu}=\int_{\mathbf{M}} \mathbf{P}_{\mathbf{x}} \mu(d \mathbf{x})$. It is then an easy matter to check that the symmetry of the $P_{t}$ 's in $L^{2}(\mu)$ translates into the statement that, for each $T \in(0, \infty)$, the processes $\{\psi(t): t \in[0, T]\}$ and $\{\psi(T-t): t \in[0, T]\}$ have the same distribution under $\mathbf{P}_{\mu}$. (In other words, $t \mapsto \psi(t)$ is reversible under $\mathbf{P}_{\mu}$.) In particular, this means that $\mathbf{P}_{\mu}$ is invariant under the natural time-shift maps on $C([0, \infty) ; \mathbf{M})$, and therefore Birkhoff's Individual Ergodic theorem applies. Thus, if $\mathbf{P}_{\mu}$ is ergodic, then, for each bounded, measurable $f: \mathbf{M} \rightarrow \mathbb{R}$,

$$
\lim _{t>\infty} \frac{1}{t} \int_{0}^{t} f(\psi(s)) d s=\langle f\rangle_{\mu} \quad \text { for } \mathbf{P}_{\mu} \text {-almost every } \psi \in C([0, \infty) ; \mathbf{M}) \text {. }
$$

Next, define the empirical distribution

$$
(t, \psi) \in(0, \infty) \times C([0, \infty) ; \mathbf{M}) \mapsto \mathbf{L}_{t}(\psi) \in \mathfrak{M}_{1}(\mathbf{M})
$$

so that

$$
\left\langle f, \mathbf{L}_{t}(\psi)\right\rangle \equiv \int_{\mathbf{M}} f(\mathbf{y}) \mathbf{L}_{t}(\psi, d \mathbf{y})=\frac{1}{t} \int_{0}^{t} f(\psi(s)) d s, \quad f \in C(\mathbf{M}) .
$$

It is then clear that (3.8) has, as an immediate corollary, the consequence that,

$$
\mathbf{L}_{t}(\psi) \Rightarrow \mu \text { as } t \nearrow \infty \text { for } \mathbf{P}_{\mu} \text {-almost every } \psi \text {. }
$$

In order to relate these considerations to the topic of the present article, we assume that $\mathfrak{5}(\mathscr{J})=\{\mu\}$ and (cf. pages $128-133$ in $[\mathrm{D} \& \mathrm{~S}]$ ) note that, for each $g \in L^{2}(\mu)$, the limit

$$
\mathscr{E}(g, g) \equiv \lim _{t>0} \frac{1}{2 t} \int_{\mathbf{M}^{2}}(g(\mathbf{y})-g(\mathbf{x}))^{2} P(t, x, d \mathbf{y}) \mu(d \mathbf{x})
$$

necessarily exists in $[0, \infty]$. In fact, when $g \in C_{0}^{1}(\mathbf{M}), \mathscr{E}(g, g)$ is nothing but $\left\langle|\nabla g|^{2}\right\rangle_{\mu}$. Thus, if $J_{\mathscr{E}}: \mathfrak{M}_{1}(\mathbf{M}) \mapsto[0, \infty]$ is defined by

$$
J_{\mathscr{\delta}}(v)= \begin{cases}\mathscr{E}\left(f^{1 / 2}, f^{1 / 2}\right) & \text { when } \quad d v=f d \mu \\ \infty & \text { if } \quad v \text { is not } \mu \text {-absolutely continuous, }\end{cases}
$$

then the logarithmic Sobolev inequality in (1.1) is equivalent to the statement that

$$
\mathbf{H}(v \mid \mu) \leqq 2 c J_{g}(v), \quad v \in \mathfrak{M}_{1}(\mathbf{M}),
$$

where

$$
\mathbf{H}(v \mid \mu) \equiv \begin{cases}\int_{\mathbf{M}} f \log f \mathrm{~d} \mu & \text { when } \quad d v=f d \mu \\ \infty & \text { if } \quad v \text { is not } \mu \text {-absolutely continuous }\end{cases}
$$


is the relative entropy of $v$ with respect to $\mu$. (Notice that the sign convention here is opposite to the usual one in physics.) In particular (cf. Theorem 5.3.10 and Exercise 5.3.16 in [D\&S]), the existence of a logarithmic Sobolev inequality for $\mu$ leads to the following more quantitative version of (3.9): for each measurable subset $\Gamma$ of $\mathfrak{M}_{1}(\mathbf{M})$,

$$
-\inf _{v \in \Gamma^{0}} J_{\mathscr{E}}(v) \leqq \lim _{t \rightarrow \infty} \frac{1}{t} \log \left[\mathbf{P}_{\mu}\left(\mathbf{L}_{t} \in \Gamma\right)\right] \leqq \varlimsup_{t \rightarrow \infty} \frac{1}{t} \log \left[\mathbf{P}_{\mu}\left(\mathbf{L}_{t} \in \Gamma\right)\right] \leqq-\inf _{v \in \bar{\Gamma}} J_{\mathscr{E}}(v),
$$

where $\Gamma^{0}$ and $\bar{\Gamma}$ are, respectively, the interior and closure of $\Gamma$ in the weak topo$\operatorname{logy}$ for $\mathfrak{M}_{1}(\mathbf{M})$.

Although the information contained in (3.11) is interesting, from a practical point of view it has serious flaw. Namely, perhaps the most significant use to which this sort of estimate might be put is that of predicting how well the Gibbs state $\mu$ can be approximated by the empirical distribution coming from a simulation based on Glauber dynamics; and presumably the reason for running such a simulation is that the measure $\mu$ is unknown. But if $\mu$ is unknown, then it is obvious that, as it stands, (3.11) has no value, since (3.11) is predicated on the assumption that $\mu$ is known well enough to use it as the initial distribution for the Glauber dynamics. Thus, before they can be considered truly useful, the estimates in (3.11) must be replaced by statements in which $\mathbf{P}_{\mu}$ is replaced by $\mathbf{P}_{\mathbf{x}}$ for arbitrary $\mathbf{x} \in \mathbf{M}$. Clearly, the problem here is a precise analogue of the one involved in the passage from the $L^{2}(\mu)$-statement in (3.3) to the uniform one in (3.5); and so it should not be surprising that the solution here is similar to the one there. In particular, we will need a path-space version of the estimate in (2.8). Thus, for each $\Lambda \in \mathbb{F}$ and $\mathbf{x} \in \mathbf{M}$, let $\mathbf{P}_{\mathbf{x}}^{\Lambda}$ be the unique probability measure on $C([0, \infty) ; \mathbf{M})$ with the property that

$$
\left(f(\psi(t))-f(\mathbf{x})-\int_{0}^{t}\left[\mathbf{A}^{\Lambda, \mathbf{x}} f\right](\psi(s)) d s, \mathscr{B}_{t}, \mathbf{P}_{\mathbf{x}}^{\Lambda}\right) \quad \text { is a mean-zero martingale }
$$

for each $f \in C_{0}^{2}(\mathbf{M})$; or, equivalently,

$$
\mathbf{P}^{\Lambda}(\psi(0)=\mathbf{x})=1 \quad \text { and } E^{\mathbf{P}_{\times}^{\Lambda}}\left[f(\psi(s+t)) \mid \mathscr{B}_{s}\right]=\left[\mathbf{P}_{t}^{\Lambda, \mathbf{x}} f\right](\psi(s)) \quad\left(\text { a.s., } \mathbf{P}_{\mathbf{x}}^{\Lambda}\right)
$$

for all $s, t \in[0, \infty)$ and $f \in C(\mathbf{M})$.

3.12 Lemma. There is a constant $C \in(0, \infty)$ with the property that

$$
\left|E^{\mathbf{P}_{y}^{A}}[F]-E^{\mathbf{P}_{x}}[F]\right| \leqq \operatorname{diam}(\mathbf{M})\left(\frac{\left(C T^{2}\right)^{N}}{N !}\right)^{1 / 2} \operatorname{Lip}(F)
$$

whenever $\Lambda \in \mathfrak{F}, \mathbf{x}, \mathbf{y} \in \mathbf{M}, T \in[1, \infty)$, and $F: C([0, \infty) ; \mathbf{M}) \rightarrow \mathbb{R}$ satisfy $\mathbf{x}_{\Lambda}=\mathbf{y}_{\Lambda}$ and

$$
|F(\hat{\psi})-F(\psi)| \leqq \operatorname{Lip}(F) \max \left\{\operatorname{dist}\left(\hat{\psi}(t)_{\mathbf{k}}, \psi(t)_{\mathbf{k}}\right): t \in[0, T] \text { and }|\mathbf{k}-\Lambda \mathrm{C}| \geqq N R\right\}
$$

for all $\psi, \psi \in C([0, \infty) ; \mathbf{M})$.

Proof. In order to prove (3.13), we will use stochastic integral equations to construct a coupling of the measures $\mathbf{P}_{\mathbf{x}}$ and $\mathbf{P}_{\mathbf{x}}^{\Lambda}$; and for this purpose, it will be convenient to think of $M$ as embedded in $\mathbb{R}^{D}$ for some sufficiently large $D \in \mathbb{Z}^{+}$. Then, taking $B=[-R, R]^{d}$, we can choose twice continuously and bounded differentiable 
functions $\sigma: \mathbb{R}^{D} \rightarrow \mathbb{R}^{D} \otimes \mathbb{R}^{D}$ and $b:\left(\mathbb{R}^{D}\right)^{B} \rightarrow \mathbb{R}^{D}$ with the property that

$$
\left[\mathbf{A}_{\mathbf{k}} f\right](\mathbf{x})=\frac{1}{2} \sum_{i, j=1}^{D}\left(\sigma \sigma^{T}\right)^{i, j}\left(x_{\mathbf{k}}\right) \frac{\partial^{2} f}{\partial x_{\mathbf{k}}^{i} \partial x_{\mathbf{k}}^{j}}(\mathbf{x})+\sum_{i=1}^{D} b^{i}\left(x_{\mathbf{k}+B}\right) \frac{\partial f}{\partial x_{\mathbf{k}}^{i}}(\mathbf{x})
$$

for all $\mathbf{k} \in \mathbb{Z}^{d}, \mathbf{x} \in \mathbf{M}$, and $f \in C^{2}\left(\left(\mathbb{R}^{D}\right)^{\mathbb{Z}^{d}}\right)$. Next, let $(\Omega, \mathscr{F}, \mathscr{W})$ be a complete probability space on which there exists a family $\left\{\beta^{\mathbf{k}}: \mathbf{k} \in \mathbb{Z}^{d}\right\}$ of mutually independent, Brownian motions $\omega \in \Omega \mapsto \beta^{\mathbf{k}}(\cdot, \omega) \in C\left([0, \infty) ; \mathbb{R}^{D}\right)$. Using the familiar techniques of Itô stochastic calculus, one can easily show that, for any non-empty $\Lambda \subseteq \mathbb{Z}^{d}$, there exists a $\mathscr{W}$-almost surely unique, $\left\{\beta^{k}: \mathbf{k} \in \mathbb{Z}^{d}\right\}$-progressively measurable $\mathbf{X}^{\Lambda}:[0, \infty) \times \mathbf{M} \times \Omega \mapsto \mathbf{M}$ such that $(t, \mathbf{x}) \in[0, \infty) \times \mathbf{M} \mapsto \mathbf{X}^{\Lambda}(t, \mathbf{x}, \omega)$ is a continuous for each $\omega \in \Omega$ and

$$
\begin{aligned}
X^{\Lambda}(T, \mathbf{x}, \omega)_{\mathbf{k}}= & x_{\mathbf{k}}+\int_{0}^{T} \sigma\left(X^{\Lambda}(t, \mathbf{x})_{\mathbf{k}}\right) d \beta^{\mathbf{k}}(t, \omega) \\
& +\int_{0}^{T} b\left(\mathbf{X}^{\Lambda}(t, \mathbf{x}, \omega)_{\mathbf{k}+B}\right) d t, \quad \mathbf{k} \in \mathbb{Z}^{d} \quad \text { and } \quad T \in[0, \infty),
\end{aligned}
$$

for $\mathscr{W}$-almost every $\omega \in \Omega$. Moreover, setting $\mathbf{X}=\mathbf{X}^{\mathbb{Z}^{d}}$ and using the martingale characterization given above, it is an elementary application of Itô's Lemma to check that the distributions of $\omega \in \Omega \mapsto \mathbf{X}^{\boldsymbol{\Lambda}}(\cdot, \mathbf{x}, \omega) \in \mathbf{M}$ and $\omega \in \Omega \mapsto \mathbf{X}(\cdot, \mathbf{x}, \omega) \in \mathbf{M}$ under $\mathscr{W}$ are, respectively, $\mathbf{P}_{\mathbf{x}}^{\Lambda}$ and $\mathbf{P}_{\mathbf{x}}$. Thus, (3.13) will follow as soon as we show that there is a $C \in(0, \infty)$ such that

$$
\begin{aligned}
& E^{\mathscr{W}}\left[\sup _{t \in[0, T]}\left|X^{\Lambda}(t, \mathbf{y})_{\mathbf{k}}-X(t, \mathbf{x})_{\mathbf{k}}\right|^{2}\right] \\
& \quad=\operatorname{diam}(M) \frac{(C T(1+T))^{N}}{N !}, \text { if } \quad \mathbf{x}_{\Lambda}=\mathbf{y}_{\Lambda} \text { and }|\mathbf{k}-\Lambda \mathrm{C}| \geqq N R .
\end{aligned}
$$

But, by elementary Itô calculus and Doob's inequality, one sees that, for any $\mathbf{k} \in \Lambda$,

$$
\begin{aligned}
& E^{\mathscr{W}}\left[\sup _{t \in[0, T]}\left|X^{\Lambda}(t, \mathbf{y})_{\mathbf{k}}-X(t, \mathbf{x})_{\mathbf{k}}\right|^{2}\right] \\
& \quad \leqq C(1+T) \int_{0}^{T} \max _{|\mathbf{j}-\mathbf{k}| \leqq R} E^{\mathscr{W}}\left[\left|X^{\Lambda}(t, \mathbf{y})_{\mathbf{j}}-X(t, \mathbf{x})_{\mathbf{j}}\right|^{2} d t, \quad T \in[0, \infty),\right.
\end{aligned}
$$

where $C \in(0, \infty)$ depends only on the $C^{1}$-bounds on the functions $\sigma$ and $b$. Hence, since $\left|X^{\Lambda}(\cdot, \mathbf{y}, \omega)_{\mathbf{k}}-X(t, \mathbf{x}, \omega)\right|$ is necessarily dominated by diam $(M)$, the desired estimate follows immediately by induction on $N \in \mathbb{Z}^{+}$.

Our next task is to develop an appropriate analogue to the estimate in Lemma 2.3.

3.14 Lemma. Assume that (1.4) holds, and set

$$
q(t)=1+e^{t / 2 c}, \quad t \in[0, \infty), \text { where } c \equiv \sup _{\substack{\Lambda \in \mathfrak{F} \\ \mathbf{x} \in \mathbf{M}}} c\left(E^{\Lambda}(\cdot \mid \mathbf{x})\right)
$$


Then, for each $\delta>0$ and all $\mathbf{x} \in \mathbf{M}, \Lambda \in \mathfrak{F}, V \in C(\mathbf{M})$, and $\Gamma \in \mathscr{B}_{\mathfrak{M}_{1}(\mathbf{M})}$,

$$
\begin{aligned}
& E^{P_{\times}^{\Lambda}}\left[e^{t\left\langle V, \mathbf{L}_{t}\right\rangle}, \mathbf{L}_{t} \in \Gamma\right] \\
& \quad \leqq e^{t \delta\|V\|_{u}} K^{|\boldsymbol{\Lambda}| / q(T)} \int_{\mathbf{M}} E^{\mathbf{P}_{y}^{\lambda}}\left[e^{t\left\langle V, \mathbf{L}_{t}\right\rangle}, \mathbf{L}_{t} \in \Gamma^{(\delta)}\right] E^{\Lambda}(d \mathbf{y} \mid \mathbf{x}), \quad x \in \mathbf{M},
\end{aligned}
$$

so long as $0<t<\infty$ with $\frac{2 T+4}{t}<\delta$. (The constant $K \in[1, \infty)$ in $(3.5)$ is the same as the one in (2.4) and the quantity $\Gamma^{(\delta)}$ denotes the open $\delta$-hull of $\Gamma$ computed with respect to the Lévy metric on $\mathfrak{M}_{1}(\mathbf{M})$.)

Proof. Note that if $\theta_{T}: C([0, \infty) ; \mathbf{M}) \rightarrow C([0, \infty) ; \mathbf{M})$ is the time-shift determined by

$$
\left[\theta_{T} \psi\right](t)=\psi(t+T), \quad(t, \psi) \in[0, \infty) \times C([0, \infty) ; \mathbf{M}),
$$

then

$$
\left\|\mathbf{L}_{t}\left(\theta_{T} \psi\right)-\mathbf{L}_{t}(\psi)\right\|_{\mathrm{var}} \leqq \frac{2 T}{t}, \quad(t, \psi) \in(0, \infty) \times C([0, \infty) ; \mathbf{M})
$$

and therefore, when $\frac{2 T+4}{t}<\delta$,

$$
E^{\mathbf{P}_{x}^{\Lambda}}\left[e^{t\left\langle V, \mathbf{L}_{t}\right\rangle}, \mathbf{L}_{t} \in \Gamma\right] \leqq e^{t \delta\|V\|_{u}} E^{\mathbf{P}_{x}^{\Lambda}}\left[e^{t\left\langle V, \mathbf{L}_{t}\right\rangle}, \mathbf{L}_{t} \circ \theta_{T+2} \in \Gamma^{(\delta)}\right]=e^{t \delta\|V\|_{u}}\left[P_{T+2}^{\Lambda, \mathbf{x}} f_{t}\right](\mathbf{x}),
$$

where

$$
f_{t}(\mathbf{y}) \equiv E^{\mathbf{P}_{\mathbf{y}}^{\Lambda}}\left[e^{t\left\langle V, \mathbf{L}_{t}\right\rangle}, \mathbf{L}_{t} \in \Gamma^{(\delta)}\right], \quad \mathbf{y} \in \mathbf{M} .
$$

Next, by (2.3), interpolation, and duality, note that, for any $q \in[2, \infty)$,

$$
\left\|P_{1}^{\Lambda, \mathrm{x}} f\right\|_{\mathrm{u}} \leqq K^{|\Lambda| / q}\|f\|_{1, \Lambda, \mathbf{x}} \text { and }\left\|P_{1}^{\Lambda, \mathbf{x}} f\right\|_{q^{\prime}, \Lambda, \mathbf{x}} \leqq K^{|\Lambda| / q}\|f\|_{1, \Lambda, \mathrm{x}}, \quad f \in C(\mathbf{M}),
$$

where $q$ ' denotes the Hölder conjugate of $q$. At the same time, by Gross's integration lemma for logarithmic Sobolev inequalities,

$$
\left\|P_{T}^{\Lambda, \mathbf{x}} f\right\|_{q(T), \Lambda, \mathbf{x}} \leqq\|f\|_{q(T)^{\prime}, \Lambda, \mathbf{x}}, \quad f \in \mathbf{C}(\mathbf{M}) ;
$$

and so (3.15) is now proved.

With these two lemmas, we now have the upper bound which we are seeking.

3.16 Lemma. Assume that (1.4) holds. Then, for each $\delta>0, V \in C_{0}(\mathbf{M})$, and $\Gamma \in \mathscr{B}_{\mathfrak{M}_{1}(\mathbf{M})}$,

$$
\varlimsup_{t \rightarrow \infty} \frac{1}{t} \log \left(\sup _{\mathbf{x} \in \mathbf{M}} E^{\mathbf{P}_{\mathbf{x}}}\left[e^{t\left\langle V, \mathbf{L}_{t}\right\rangle}, \mathbf{L}_{t} \in \Gamma\right]\right) \leqq \delta\|V\|_{\mathbf{u}}+\varlimsup_{t \rightarrow \infty} \frac{1}{t} \log \left(E^{\mathbf{P}_{\mu}}\left[e^{t\left\langle V, \mathbf{L}_{t}\right\rangle}, \mathbf{L}_{t} \in \Gamma^{(\delta)}\right]\right) .
$$

In particular,

$$
\varlimsup_{t \rightarrow \infty} \frac{1}{t} \log \left[\sup _{\mathbf{x} \in \mathbf{M}} \mathbf{P}_{\mathbf{x}}\left(\mathbf{L}_{t} \in \Gamma\right)\right] \leqq-\inf _{v \in \bar{\Gamma}} J_{\mathscr{E}}(v)
$$

Proof. Given (3.17) and (3.11), (3.18) becomes an easy application of the fact (cf. Exercise 5.3 .16 in $[\mathrm{D} \& \mathrm{~S}]$ ) that $(3.10)$ implies $J_{\mathscr{E}}$ is lower semi-continuous. To 
prove (3.17), note that, for each $\delta>0$, there exist an $A \in \mathfrak{F}$ and an $L \in[0, \infty)$ (depending only on $\delta$ and $V$ ) such that, for each $t \in(0, \infty)$, one can construct functions $F_{t}$ and $G_{t}$ on $C([0, \infty) ; \mathbf{M})$ with the properties that

$$
\begin{aligned}
e^{t\left\langle V, \mathbf{L}_{t}(\psi)\right\rangle} \mathbf{1}_{\Gamma}\left(\mathbf{L}_{t}(\psi)\right) & \leqq F_{t}(\psi) \leqq e^{t\left\langle V, \mathbf{L}_{t}(\psi)\right\rangle} \mathbf{1}_{\Gamma^{(\delta / 2)}}\left(\mathbf{L}_{t}(\psi)\right) \\
& \leqq G_{t}(\psi) \leqq e^{t\left\langle V, \mathbf{L}_{t}(\psi)\right\rangle} \mathbf{1}_{\Gamma^{(\delta)}}\left(\mathbf{L}_{t}(\psi)\right)
\end{aligned}
$$

for all $\psi \in C([0, \infty) ; \mathbf{M})$, and

$\left|F_{t}(\hat{\psi})-F_{t}(\psi)\right| \vee\left|G_{t}(\hat{\psi})-G_{t}(\psi)\right| \leqq L e^{t\|V\|_{\mathrm{u}}} \max \left\{\operatorname{dist}\left(\hat{\psi}(s)_{\mathbf{j}}, \psi(s)_{\mathbf{j}}\right): s \in[0, t]\right.$ and $\left.\mathbf{j} \in A\right\}$ for all $\psi, \hat{\psi} \in C([0, \infty) ; \mathbf{M})$. Hence, if $\Lambda(t) \equiv\left\{\mathbf{k} \in \mathbb{Z}^{d}:|\mathbf{k}-A| \leqq t^{3}\right\}, \quad t \in[1, \infty)$, then, by (3.13) and (3.15),

$$
\begin{aligned}
E^{\mathbf{P}_{\mathbf{x}}}\left[e^{t\left\langle V, \mathbf{L}_{t}\right\rangle}, \mathbf{L}_{t} \in \Gamma\right] & \leqq E^{\mathbf{P}_{\mathbf{y}}^{\Lambda(t)}}\left[F_{t}\right]+L\left(\frac{C}{t}\right)^{t^{3 / 2}} e^{t\|\boldsymbol{V}\|_{\mathrm{u}}} \\
& \leqq e^{t \delta\|V\|_{u}} K^{(2|\Lambda(t)|) /(q(\sqrt{ } t))} \int_{\mathbf{M}} E^{\mathbf{P}_{z}^{\Lambda}(t)}\left[G_{t}\right] E^{\Lambda(t)}(d \mathbf{z} \mid \mathbf{y})+L\left(\frac{C}{t}\right)^{t^{3 / 2}} e^{t\|\boldsymbol{V}\|_{\mathrm{u}}} \\
& \leqq e^{t \delta\|V\|_{u}} K^{(2|\Lambda(t)|) /(q(\sqrt{ } t))} \int_{\mathbf{M}} E^{\mathbf{P}_{\mathbf{z}}}\left[G_{t}\right] E^{\Lambda(t)}(d \mathbf{z} \mid \mathbf{y})+2 L\left(\frac{C}{t}\right)^{t^{3 / 2}}
\end{aligned}
$$

for all sufficiently large $t \in[0, \infty)$ and all $\mathbf{x}, \mathbf{y} \in \mathbf{M}$ satisfying $\mathbf{y}_{\Lambda(t)}=\mathbf{x}_{\Lambda(t)}$. But the final expression in the preceding only depends on $\mathbf{y}_{\boldsymbol{\Lambda}(t) \mathrm{c}}$, and so, after integrating it with respect to $\mu$, we obtain

$$
E^{\mathbf{P}_{\mathbf{x}}}\left[e^{t\left\langle V, \mathbf{L}_{t}\right\rangle}, \mathbf{L}_{t} \in \Gamma\right] \leqq e^{t \delta\|V\|_{\mathrm{u}}} K^{(2|\Lambda(t)|) /(q(\sqrt{t}))} E^{\mathbf{P}_{\mu}}\left[e^{t\left\langle V, \mathbf{L}_{t}\right\rangle}, \mathbf{L}_{t} \in \Gamma^{(\delta)}\right]+2 L\left(\frac{C}{t}\right)^{t^{3 / 2}} e^{t\|V\|_{\mathrm{u}}}
$$

for all $\mathbf{x} \in \mathbf{M}$ and sufficiently large $t \in[1, \infty)$; and clearly (3.17) follows immediately from this.

3.19 Theorem. Assume that (1.4) holds. Then, for each $\Gamma \in \mathscr{B}_{\mathfrak{M}_{1}(\mathbf{M})}$ :

$$
\begin{aligned}
-\inf _{v \in \Gamma^{0}} J_{\mathscr{E}}(v) & \leqq \lim _{t \rightarrow \infty} \frac{1}{t} \log \left[\inf _{x \in \mathbf{M}} \mathbf{P}_{\mathbf{x}}\left(\mathbf{L}_{t} \in \Gamma\right)\right] \\
& \leqq \varlimsup_{t \rightarrow \infty} \frac{1}{t} \log \left[\sup _{x \in \mathbf{M}} \mathbf{P}_{\mathbf{x}}\left(\mathbf{L}_{t} \in \Gamma\right)\right] \leqq-\inf _{v \in \bar{\Gamma}} J_{\mathscr{E}}(v) .
\end{aligned}
$$

In particular, for any continuous $\Phi: \mathfrak{M}_{1}(\mathbf{M}) \rightarrow \mathbb{R}$ with the property that

$$
\begin{gathered}
\sup _{\substack{t \in(0, \infty) \\
\mathbf{x} \in \mathbf{M}}} E^{\mathbf{P}_{\mathbf{x}}}\left[e^{(1+\varepsilon) t \Phi\left(\mathbf{L}_{t}\right)}\right]^{1 / t}<\infty \quad \text { for some } \varepsilon>0, \\
\varlimsup_{t \rightarrow \infty} \sup _{\mathbf{x} \in \mathbf{M}}\left|\frac{1}{t} \log \left(E^{\mathbf{P}_{\mathbf{x}}}\left[e^{t \Phi\left(\mathbf{L}_{t}\right)}\right]-\sup _{v \in \mathfrak{M}_{\mathbf{1}}(\mathbf{M})}\left(\Phi(v)-J_{\mathscr{E}}(v)\right)\right)\right|=0 .
\end{gathered}
$$

Proof. The upper bound in (3.20) is already contained in (3.18), and the passage from (3.20) to (3.21) is an application of Varadhan's Laplace asymptotic lemma (cf. Theorem 2.1.10 in [D\&S]). Thus, all that remains is to prove the lower bound 
in (3.20). That is, we must show that

$$
\varliminf_{r \succ 0}{\underset{\lim }{t \rightarrow \infty}}_{t} \frac{1}{t} \log \left[\inf _{\mathbf{x} \in \mathbf{M}} \mathbf{P}_{\mathbf{x}}\left(\mathbf{L}_{t} \in B(v, r)\right)\right] \geqq-J_{\mathscr{E}}(v), \quad v \in \mathfrak{M}_{1}(\mathbf{M}),
$$

where $B(v, r)$ denotes the Lévy ball of radius $r$ around $v$ in $\mathfrak{M}_{1}(\mathbf{M})$. In fact, by the same sort of argument as the one given to check the corresponding assertion in the proof of Theorem 5.3.11 in [D\&S], we need only consider $v \in \mathfrak{M}_{1}(\mathbf{M})$ of the form $d v=f d \mu$, where $f$ is a uniformly positive element of $C_{0}^{2}(\mathbf{M})$. Given such a $v$, set $u=f^{1 / 2}, V=-\frac{\mathbf{A} u}{u}$,

$$
R_{t}(\psi)=\frac{u(\psi(t))}{u(\psi(0))} \exp \left[\int_{0}^{t} V(\psi(s)) d s\right], \quad(t, \psi) \in[0, \infty) \times C([0, \infty) ; \mathbf{M}),
$$

and note that, because $\left(R_{t}, \mathscr{B}_{t}, \mathbf{P}_{\mathbf{x}}\right)$ is a martingale, there is a unique element $\mathbf{Q}_{\mathbf{x}}$ of $\mathfrak{M}_{1}(C([0, \infty) ; \mathbf{M}))$ such that

$$
\mathbf{Q}_{\mathbf{x}}(\Gamma)=E^{\mathbf{P}_{\mathbf{x}}}\left[R_{t}, \Gamma\right] \text { for all } t \in[0, \infty) \text { and } \Gamma \in \mathscr{B}_{t} .
$$

Moreover (cf. Lemmas 5.3.5 and 5.3.9 in [D\&S]),

$$
\lim _{t \rightarrow \infty} \mathbf{Q}_{\mu}\left(\mathbf{L}_{t} \in B(v, r)\right)=1 \text { for all } r \in(0, \infty)
$$

and therefore, by (3.17), we see that

$$
\varliminf_{r \searrow 0} \varliminf_{t \rightarrow \infty} \inf _{\mathbf{x} \in \mathbf{M}} \mathbf{Q}_{\mathbf{x}}\left(\mathbf{L}_{t} \in B(v, r)\right)=1 .
$$

Hence, since

$$
\mathbf{P}_{\mathbf{x}}\left(\mathbf{L}_{t} \in B(v, r)\right) \geqq \varepsilon e^{-t M(r)} \mathbf{Q}_{\mathbf{x}}\left(\mathbf{L}_{t} \in B(v, r)\right),
$$

where

$$
\varepsilon \equiv \inf \left\{\frac{u(\mathbf{y})}{u(\mathbf{x})}: \mathbf{x}, \mathbf{y} \in \mathbf{M}\right\} \text { and } M(r)=\sup \{\langle V, \hat{v}\rangle: \hat{v} \in B(v, r)\},
$$

we conclude that

$$
\begin{aligned}
& \varliminf_{r \searrow 0} \varliminf_{t \rightarrow \infty} \frac{1}{t} \log \left[\inf _{\mathbf{x} \in \mathbf{M}} \mathbf{P}_{\mathbf{x}}\left(\mathbf{L}_{t} \in B(v, r)\right)\right] \geqq-\lim _{r \searrow 0} M(r) \\
& =\int_{\mathbf{M}} \frac{\mathbf{A} u}{u} d v=\int_{\mathbf{M}} u \mathbf{A} u d \mu=-J_{\mathscr{E}}(v) .
\end{aligned}
$$

\section{References}

[A\&H] Aizenman, M., Holley, R.: Rapid convergence to equilibrium of stochastic Ising Models in the Dobrushin Shlosman régime, Percolation Theory and Ergodic Theory of Infinite Particle Systems, H. Kesten, (ed.), IMS Volumes in Math. and Appl. vol. 8, pp. 1-11. Berlin, Heidelberg, New York: Springer 1987 
[D] Dobrushin, R.: Markov processes with a large number of locally interacting components-Existence of the limiting process and its ergodicity. Probl. Peredaci Inform. 7, 70-87 (1971)

[D\&S] Deuschel, J.-D., Stroock, D.: Large Deviations, Pure and Appl. Math. Series, vol. 137. Boston: Academic Press 1989

[Dob\&S, 1] Dobrushin, R.L., Shlosman, S.B.: Constructive criterion for the uniqueness of Gibbs field, Statistical Physics and Dynamical Systems, Rigorous Results, pp. 347-370. Fritz, Jaffe, and Szasz (eds.). Basel-Boston: Birkhauser 1985

[Dob\&S, 2] - Completely analytical Gibbs fields. Statistical Physics and Dynamical Systems, Rigorous Results, pp. 371-403. Fritz, Jaffe, and Szasz, (eds.), Basel-Boston: Birkhäuser 1985

[Dob\&S, 3] — Completely analytical interactions: Constructive description. J. Stat. Phys. 46, 983-1014 (1987)

[F] Federbush, P.: Partially alternative derivation of a result of Nelson. J. Math. Phys. 10 (1), 50-52 (1989)

[G,1] Gross, L.: Logarithmic Sobolev inequalities. Am. J. Math. 97, 553-586 (1979)

$[\mathrm{G}, 2] \longrightarrow$ : Absence of second-order phase transitions in the Dobrushin uniqueness region. J. Stat. Phys. 25 (1), 57-72 (1981)

[H] Holley, R.: Possible rates of convergence in finite range, attractive spin systems. Contemp. Math. 41, 215-234 (1985)

[H\&S, 1] Holley, R., Stroock, D.: Applications of the stochastic Ising Model to the Gibbs states. Commun. Math. Phys. 48, 249-265 (1967)

[H\&S, 2] - Uniform and $L^{2}$ convergence in one dimensional stochastic Ising models. Commun. Math. Phys. 123, 85-93 (1989)

[L] Liggett, T.: Infinite Particle Systems. Grundlehren Series, vol. 276, Berlin, Heidelberg, New York: Springer 1985

[S\&Z] Stroock, D., Zegarlinski, B.: The logarithmic Sobolev inequality for lattice gases with continuous spins. J. Funct. Anal. (to appear)

Communicated by M. Aizenman 
